\title{
Inhibitory Units: An Organizing Nidus for Feature-Selective SubNetworks in Area V1
}

\author{
Ganna Palagina, ${ }^{1,2}$ Jochen F. Meyer, ${ }^{3}$ and $\mathbb{D S}^{-S t e l i o s}$ M. Smirnakis ${ }^{1,2,3}$ \\ ${ }^{1}$ Department of Neurology, Brigham and Women's Hospital, Harvard Medical School Boston, Massachusetts 02115, ${ }^{2}$ Jamaica Plain Veterans \\ Administration Hospital, Boston, Massachusetts 02130, and '3Baylor College of Medicine, Houston, Texas 77030
}

Neuronal circuits often display small-world network architecture characterized by neuronal cliques of dense local connectivity communicating with each other through a limited number of cells that participate in multiple cliques. The principles by which such cliques organize to encode information remain poorly understood. Similarly tuned pyramidal cells that preferentially target each other may form multicellular encoding units performing distinct computational tasks. The existence of such units can reflect upon both spontaneous and stimulus-driven population events.

We applied two-photon calcium imaging to study spontaneous population bursts in layer 2/3 of area V1 in male C57BL/6 mice. To identify potential small-world cliques, we searched for pyramidal cells whose calcium events had a consistent temporal relationship with the events of local inhibitory interneurons. This was guided by the intuition that groups of neurons whose synchronous firing represents a temporally coherent computational unit should be inhibited together. Pyramidal members of these interneuron-centered clusters on average displayed stronger functional connectivity between each other than with nonmember pyramidal neurons. The structure of the clusters evolved during postnatal development: cluster size and overlap between clusters decreased with developmental maturation. Pyramidal neurons in a cluster showed higher than chance tuning function similarity between each other and with the linked interneuron. Thus, spontaneous population events in V1 are shaped by small-world subnetworks of pyramidal neurons that share functional properties and work as a coherent unit with a local interneuron. These interneuron-pyramidal cell partnerships may represent a fundamental neocortical unit of computation at the population level.

Key words: interneuron-pyramidal cell clusters; modularity; multineuronal bursts; neuronal ensembles; small world; spontaneous activity

Significance Statement

Neuronal circuit in layer 2/3 of mouse area V1 possesses small-world network architecture, where cliques of densely interconnected neurons ("small worlds") communicate via restricted number of hub cells. We show that: (1) in mouse V1 individual small-world cliques preferably incorporate pyramidal neurons with similar visual feature tuning, and (2) ongoing population activity of such pyramidal neuron clique is temporally linked to the activity of the local interneuron sharing its feature tuning with the clique members. Functional grouping of similarly tuned interneurons and pyramidal cells into cliques may ensure that ensembles of functionally alike pyramidal cells recruited during perceptual tasks and spontaneous activity are also turned off together as a unit, with interneurons serving as organizers of linked pyramidal ensemble activity.

\section{Introduction}

Sensory stimuli are encoded by the joint firing of neuronal groups rather than isolated neurons (Uhlhaas et al., 2009; Buzsáki, 2010).

\footnotetext{
Received Aug. 28, 2018; revised March 28, 2019; accepted April 1, 2019.

Author contributions: G.P. and S.M.S. designed research; G.P. and J.F.M. performed research; G.P. and J.F.M. analyzed data; G.P. wrote the paper.

This work was supported a Howard Hughes Medical Institute Early Career Award to S.M.S., the March of Dimes Foundation, the Simons Foundation, and National Eye Institute Grant R01-EY-109272. We thank Ryan Ash for helpful comments, Sergey Torsky for excellent technical assistance, and Dmitry Yatsenko and Andreas Tolias for sharing MATLAB code for the motion correction algorithm.

The authors declare no competing financial interests.
}

The principles by which neuronal groups organize to encode information remain poorly understood. A leading hypothesis is that similarly tuned pyramidal cells (PCs) form multicellular encoding units yoked to a similar purpose. Indeed, functional and anatomical connectivity in the neocortex is nonrandom (Song et al., 2005). Visual cortex shows specificity of synaptic connections: strongest synapses are formed between PCs with similar visual

Correspondence should be addressed to Ganna Palagina at gpalagina@bwh.harvard.edu. https://doi.org/10.1523/JNEUROSCI.2275-18.2019

Copyright $\odot 2019$ the authors 
response properties (Yoshimura et al., 2005; Ko et al., 2011; Wertz et al., 2015; Lee et al., 2016). Excitatory synaptic drive of a $\mathrm{PC}$ is thus dominated by few strong inputs provided by neurons sharing stimulus preference, such as receptive field structure (Cossell et al., 2015). Moreover, connection specificity also extends to fast-spiking parvalbumin-positive interneurons. Although these interneurons densely innervate most PCs in their vicinity (Packer and Yuste, 2011), the strength of their allcovering inhibitory drive is highly variable across target cells. The strongest inputs of a specific parvalbumin-positive interneuron go to PCs providing strongest reciprocal excitation to this interneuron (Yoshimura and Callaway, 2005; Znamenskiy et al., 2018). Thus, despite salt-and-pepper organization of visual feature tuning (Kalatsky and Stryker, 2003; Ohki et al., 2005), rodent V1 may still harbor functional modules based on visual responsespecific synaptic connectivity.

Functional specificity of synaptic connections should reflect on the spatiotemporal profile of spontaneous population events in the neocortex. We used two-photon calcium imaging to study spontaneous population events in layer $2 / 3$ of mouse area V1 during postnatal maturation [postnatal day (P)8-P52]. Throughout this period both size and duration of spontaneous population bursts formed scale-free distributions obeying a power law. The same was true for the degree of "functional connectivity", a measure of pairwise synchrony across cells. These observations are consistent with a hierarchical small-world-net architecture, characterized by cliques of cells with dense local connectivity ("small worlds") sparsely connecting to each other via a restricted number of cells with unusually high count of functional links (Luce and Perry, 1949; Watts and Strogatz, 1998; Bonifazi et al., 2009; Sporns, 2011). Small-world architecture (Watts and Strogatz, 1998; Lago-Fernández et al., 2000) combines local segregation with short average path length between individual nodes resulting in faster signal propagation at low wiring cost. Segregation of neuronal modules within the small-world network may increase the speed and efficiency of local computations (such as visual feature detection) while short paths between individual modules provide a means to fast integration of local information to support global processes involving synchronization of many modules.

Cortical interneurons innervate a significant percentage of local PCs (Fino and Yuste, 2011; Packer and Yuste, 2011) and therefore provide a natural way to organize and control local pyramidal cliques (Bonifazi et al., 2009; Cossart, 2014), whose members need to fire coherently to process information. To identify candidate small-world groups we searched for cells whose calcium events had a consistent temporal relationship to events recorded from local interneurons. This was guided by the intuition that groups of neurons whose synchronous firing represents a temporally coherent computational unit (or feature) ought to be inhibited together. We thus identified clusters of pyramidal neurons whose firing is temporally linked to the firing of one or more local interneurons. These clusters did not remain static during postnatal development: both cluster size and overlap with other clusters decreased over time as pyramidal neurons became progressively more selective, "linking" to fewer neighboring interneurons. Notably, pyramidal neurons in a cluster show higher tuning function similarity than expected, with each other and with their linked interneurons. Our findings suggest that spontaneous population events in the visual cortex are shaped by smallworld modular networks of pyramidal neurons that share functional properties and work as a temporally coherent unit with one or more local interneurons. We argue that such groups represent a fundamental neocortical unit of computation at the population level.

\section{Materials and Methods}

Animals and surgery. All experimental procedures were performed in accordance with institutional and federal animal welfare guidelines and were approved by the institutional animal care and use committee. We used male C57BL/6 mice expressing Td-Tomato in Dlx5/6-positive interneurons (Monory et al., 2006; Madisen et al., 2010). The mice were produced by crossing the Ai9 mice (The Jackson Laboratory, B6.CgGt(ROSA)26Sor ${ }^{\text {tm9 } 9(C A G-t d T o m a t o) H z e} / \mathrm{J}$, stock 007909), carrying a targeted insertion into the Gt(ROSA)26Sor locus with a loxP-flanked STOP cassette preventing transcription of a CAG promoter-driven red fluorescent protein variant (tdTomato; Madisen et al., 2010), and Dlx5/6-Cre mice, carrying a Cre-recombinase gene, expressed under the zebrafish Dlx5/6 regulatory sequences (Monory et al., 2006; The Jackson Laboratory, $\operatorname{Tg}(\mathrm{dlx} 5 \mathrm{a}$-cre $) 1 \mathrm{Mekk} / \mathrm{J}$, stock 008199). The expression of Cre in these mice follows the profile of expression of native mouse Dlx 5 homeobox gene during interneuronal specification in ganglionic eminences and subsequent migration to the cortex. The expression then persists into adulthood in hippocampal and neocortical interneurons (Stühmer et al., 2002; Monory et al., 2006). In the hippocampus and the neocortical layers 1 and $2 / 3$, the expression of Cre in the interneurons is near-universal: it is present in 93-100\% GABA-positive cells (Stühmer et al., 2002; Monory et al., 2006). In the neocortex all major interneuron subtypes express Cre: $\sim 100 \%$ of parvalbumin-positive cells, $>90 \%$ of calretininpositive cells, and $\sim 100 \%$ of nNOS-expressing and NPY-expressing cells (Stühmer et al., 2002). Thus, offspring mice, carrying both the floxstopped td-tomato and Cre, express Td-Tomato in all neocortical interneurons originating from medial and caudal ganglionic eminences (Monory et al., 2006; Rossignol, 2011).

In addition to the above information, we checked the level of Crerecombination (by Td-tomato labeling) in the V1 of Dlx5/6-Cre $\times$ Ai9 cross (used in the study) ourselves, using as a reference recombination data from Viaat-Cre $\times$ Ai9 cross (Chao et al., 2010). For Viaat-Cre line (Chao et al., 2010) there exists data specifically describing cortical recombination level ( $\sim 98 \%$ of cortical interneurons of all types). We recorded and compared 10 layer 2/3 fields-of-view (FOVs) from Viaat-Cre $\times$ Ai9 cross mouse ( 2 mice, 5 FOV per mouse at 150-250 $\mu \mathrm{m}$ depth, corresponding to layer $2 / 3$ ) and 10 layer $2 / 3$ FOVs from Dlx $5 / 6$-Cre $\times$ Ai9 cross mouse ( 2 mice, 5 FOV per mouse at $150-250 \mu \mathrm{m}$ depth). We found that F1 Dlx5/6 $\times$ Ai9 animals had similar density of Td tomato labeled neurons as F1 Viaat-Cre $\times$ Ai9 animals in layer $2 / 3$ of area V1: specifically, Dlx5/6-Cre $\times$ Ai9 had $488.7 \pm 25.4$ (mean \pm SEM) Td-tomato labeled cells per $\mathrm{mm}^{2}(n=10 \mathrm{FOVs})$ compared with $491 \pm 36.2$ cells per $\mathrm{mm}^{2}(n=10$ FOVs $)$ for Viaat-Cre $\times$ Ai9 animals. This suggests that essentially all ( $\sim 98 \%)$ of area V1 2/3 interneurons are labeled with $\mathrm{Td}$ tomato in Dlx5/6-Cre $\times$ Ai9 mice, in agreement with Stühmer et al. (2002) and Monory et al. (2006).

Mice were reared in a $12 \mathrm{~h}$ dark/light cycle until P8-P52 and subsequently were used for recordings. During surgery, mice where anesthetized with $2 \%$ isoflurane, which was delivered in pure oxygen via tubing near the nose of the animal. Local anesthesia with lidocaine (2\%) was given under the skin and on the skull. We placed a $3 \mathrm{~mm}$ round craniotomy above V1 and pressure injected 1 mM OGB-1 with $100 \mu$ How $\mathrm{M}$ SR-101 dissolved in Pluronic-F127 via a glass pipette $200 \mu \mathrm{m}$ below the dura (Stosiek et al., 2003). All injection sites were located 2-3 mm lateral from midline and $1-1.5 \mathrm{~mm}$ frontal to the transverse sinus, placing them in visual cortex (Wang and Burkhalter, 2007). After injection, we fixed a glass coverslip above V1 with Vetbond glue. We kept eyes moisturized using a topical eye ointment (polydimethylsiloxane-200, SigmaAldrich). The isoflurane level during recordings was maintained at $0.6 \%$ in adult animals. Total dataset consists of 21 FOVs from 18 animals (6 animals per age group).

Two-photon imaging. We used a Prairie Ultima-IV two-photon microscope with custom modifications, fed by a Chameleon Ti:sapphire Ultra-II laser and equipped with two Hamamatsu photomultiplier tubes. Prairie View software v4.1.1.4 was used to control the laser and collect images (Prairie Technologies). We imaged cells 120-200 $\mu \mathrm{m}$ below the 
pia, in layer $2 / 3$ of mouse V1. The laser was set at a wavelength of $820 \mathrm{~nm}$. At this wavelength, red fluorescing cells were either Td-Tomatoexpressing interneurons or SR-101-stained astrocytes. We selected an FOV containing 100-320 cells for imaging and acquired images using a $20 \times$ objective lens ( 0.95 NA water-immersion, Olympus) at acquisition speeds ranging from 3.2 to $11 \mathrm{~Hz}$, depending on FOV size. To separate interneurons from astrocytes we examined their morphology (astrocytes have distinct visible processes), shape, and frequency of their calcium events, and the presence of red Td-tomato fluorescence before the injection of OGB-SR-101 mixture (when available). Most interneurons recorded from adult animals also showed strong visual responses to drifting gratings.

Visual stimulation. We constructed visual stimuli using the MATLAB Psychophysics Toolbox (http://www.psychtoolbox.org). We used drifting square-wave gratings with a temporal frequency of $2 \mathrm{~Hz}$ and a spatial frequency of 0.05 cycles $/^{\circ}$. The gratings were full-field and covered $60^{\circ} \times$ $80^{\circ}$ in the contralateral visual space. Each visual presentation trial lasted $5 \mathrm{~s}: 2 \mathrm{~s}$ of visual stimulus presentation, followed by $3 \mathrm{~s}$ of spatially uniform illumination. We kept mean luminance constant throughout both the background and the stimulation periods. We presented stimuli on a flat LCD monitor (Dell), which was located $27 \mathrm{~cm}$ from the mouse eye, and covering $60^{\circ} \times 80^{\circ}$ in the contralateral monocular visual field. Presentation was monocular. We covered the non-stimulated eye with ointment and black foil during the experiment (Palagina et al., 2017).

Patch-clamp recordings. Whole-cell and loose-patch recordings were obtained with a Heka EPC-10 USB amplifier in current-clamp mode using standard techniques (Margrie et al., 2002). Six to $8 \mathrm{M} \Omega$ glass pipettes filled with an intracellular solution (in mM: $105 \mathrm{~K}$-gluconate, 30 $\mathrm{KCl}, 10 \mathrm{HEPES}, 10$ phosphocreatine, $4 \mathrm{ATP}-\mathrm{Mg}$, and $0.3 \mathrm{GTP}$, adjusted to $290 \mathrm{mOsm}$ and $\mathrm{pH} 7.3$ with $\mathrm{KOH}$, containing $10 \mu \mathrm{M}$ AlexaFluor-594 or tetramethylrhodamine dextran; Invitrogen) were advanced under two-photon visual guidance, initially with $\sim 100$ mbar pressure, then $\sim 40$ mbar when the tip reached $\sim 50 \mu \mathrm{m}$ under the dura. We reduced pressure to $\sim 20 \mathrm{mbar}$ when approaching a cell. Once resistance increased to $\sim 150 \%$ of the initial value, laser scanning was stopped and up to $200 \mathrm{mbar}$ negative pressure was applied, until the resistance increased up to $200 \mathrm{M} \Omega$. When successful, a gigaohm seal was typically formed within $2 \mathrm{~min}$. The pipette was retracted carefully by a few micrometers to avoid penetration of the interior compartments of the cell during breakin. Then, $\sim 200 \mathrm{~ms}$ pulses of negative pressure (starting at $10 \mathrm{psi}$ and increasing gradually) were applied via a Picospritzer with a vacuum module until the patch of membrane was broken. Fast pipette capacitance was compensated before break-in, and slow capacitance was compensated afterward.

\section{Experimental design and statistical analysis}

Eighteen male C57BL/6 mice expressing Td-Tomato in Dlx5/6-positive interneurons were used in the study. The mice were divided into three age groups ( $n=6$ animals per group): before eye opening ( $<\mathrm{EO}), 8$ - to 10-d-old (P8-P10); EO (eye opening) period, between 12- and 16-d-old (P12-P16), and adult animals, older than $34 \mathrm{~d}$ (P35-P52). In each animal, we imaged 1-2 FOVs to simultaneously collect spontaneous and stimulus-evoked calcium transients from all neurons in the FOV (78292 neurons at a time, on average 148 neurons per FOV). Summarily, we recorded 10-40 min of spontaneous activity per FOV (total of $n=21$ FOVs, $n=7$ FOVs per age group). In each FOV we identified spontaneous population bursts and performed small-world network analysis (described in detail in the sections Population bursts and Small-world network parameters and analysis). We next identified interneuron-PC partnerships [interneuron pyramidal partner (IPP) clusters; described in section Identifying IPPs], and described functional connectivity within these partnerships (see Global functional connectivity of the PCs and group correlation strength of the IPP clusters). We identified and used for the analyses described in sections "Identifying interneuron pyramidal partners", "Global functional connectivity of the PCs and group correlation strength of the IPP clusters" and "Global functional connectivity of the PCs during multineuronal bursts" $n=38$ IPP clusters for the adult group, $n=41$ IPP clusters for the eye-opening group, and $n=45$ IPP clusters for the group before eye opening. On average, each FOV contained six IPP clusters.
Population bursts: extraction of calcium events' onsets and definition of the burst-related activity. We typically recorded 10-40 min of spontaneous activity per FOV (1-4 movies). The acquired raw fluorescence movies were motion-corrected using cross-correlation between subsequent frames of the movies containing red (Td-tomato- and SR-101-based) fluorescent signals. This allowed us to remove slow $x-y$ plane drifts from the movies. After motion correction, we used ImageJ software (Abramoff et al., 2004) to draw the ROIs of cells around cell body centers, staying $1-2$ pixels $(\sim 2.4 \mu \mathrm{m})$ from the margin of a cell to avoid contamination from neuropil signals. We then extracted centers of mass of the ROIs, averaged the signals of cell ROI pixels and converted them to dF/F. To determine the onsets of OGB-1 spontaneous calcium responses, the dF/F time course for each cell was thresholded, using the noise portion of the data, to 3 standard deviations (SD) above baseline. We applied moving average filter ( $3 \mathrm{~s}$ window) to the $\mathrm{dF} / \mathrm{F}$ and then subtracted the result from the $\mathrm{dF} / \mathrm{F}$ to obtain the noise and calculate the individual threshold for each cell. We used current-clamp recordings in three cells to verify that the above procedure was faithfully detecting the onsets of actual action potential bursts. We aligned the calcium event onset series with corresponding current-clamp recordings. Then, the $4 \mathrm{~s}$ periods after each event onset, corresponding to the rise and decay of the calcium transient, were taken out of the current-clamp recording, and the remainder of it was used for baseline calculations. We then determined whether any frames during and $2 \mathrm{~s}$ after event onset frame contained action potentials, and how many. The $2 \mathrm{~s}$ window encompasses the fast rise and peak area of calcium event, where most action potentials occur (Vogelstein et al., 2010). We determined the median spike rate in each $2 \mathrm{~s}$ window across all events, and determined whether any individual frame in this window contained $>1$ action potential (doublets and multiplets). Then we broke the baseline portion of the recording into $2 \mathrm{~s}$ intervals, and repeated this procedure for those intervals. Seventy-seven percent of calcium events detected by this method corresponded to doublets, triplets, etc., of action potentials, whereas single action potentials were caught in only $23 \%$ of cases. In contrast, the parts of the recordings that did not have detected events (baseline) consisted mainly of solitary action potentials; doublets and multiplets appeared in only $35 \%$ of baseline trials. In addition to this, the action potential packets detected by our procedure contained larger spike count than those belonging to baseline trials: median spike rate was $3.25 \mathrm{~Hz}$ for event trials compared with $0.5 \mathrm{~Hz}$ for baseline trials. This shows that our procedure discriminates in favor of cellular events reflecting reverberating recurrent population activity (population bursts), as the detected cellular events mostly correspond to bursts of action potentials occurring at higher rate, as opposed to sparser solitary spikes observed during baseline periods.

Detected cellular event onsets were then used to form an eventogram for each FOV movie. This initial eventogram was used to assess functional connectivity of the neurons in the FOV (by using pairwise Pearson correlation between their event trains) and to calculate basic network properties such as small-world coefficient, clustering coefficient and average path length (Fig. 1). Two types of events were found in the initial eventogram: multineuronal population bursts and solitary cellular bursts. We defined multineuronal population bursts as periods of time when two or more cells in the FOV had onset of calcium responses in one or more consecutive frames, without skipping a frame. The first frame of a multineuronal population burst is preceded by a frame that is empty. Thus, multineuronal population burst has size of two cells or larger. The multineuronal burst continues until a frame is encountered containing no calcium event onsets in any of the FOV's cells. Burst duration is a total time between first and last frames of the burst. Solitary bursts are calcium events observed in a single cell and invariably have a size of 1 . To focus further analysis on groups of cells that are coactive during multineuronal bursts, we excluded solitary cell bursts from the eventogram, so that only multineuronal population burst activity was used in the analysis of potential interneuron-PC partnerships and characterization of those partnerships (see Figs. 2-5).

Small-world network parameters and analysis. Determination of "linked cells" for small-world analysis was made based on presence of significant pairwise linear Pearson correlation between cells. To determine whether the Pearson correlation coefficient in each pair was signif- 
A
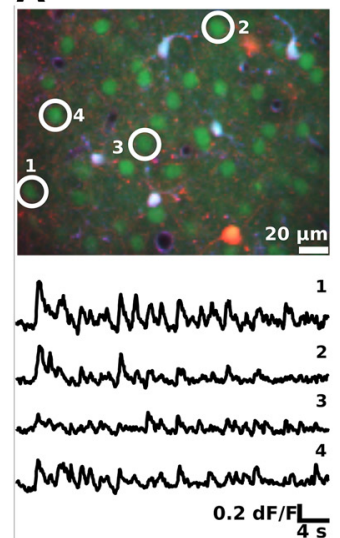

C

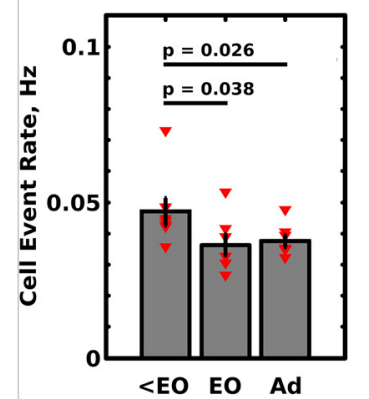

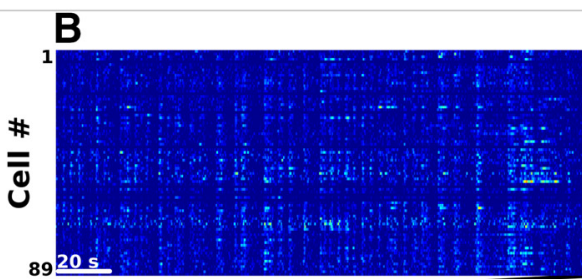
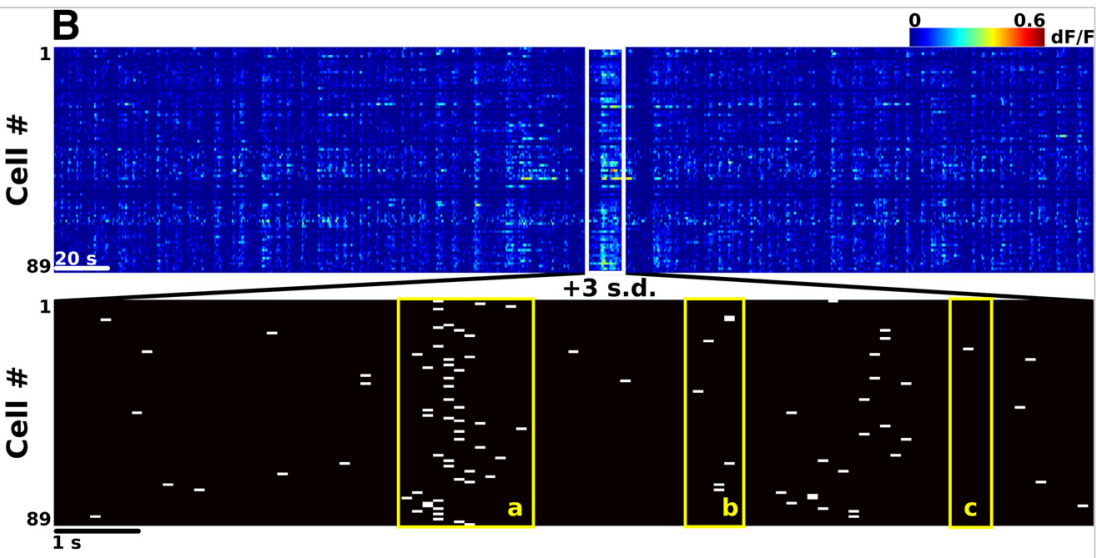

+3 s.d.
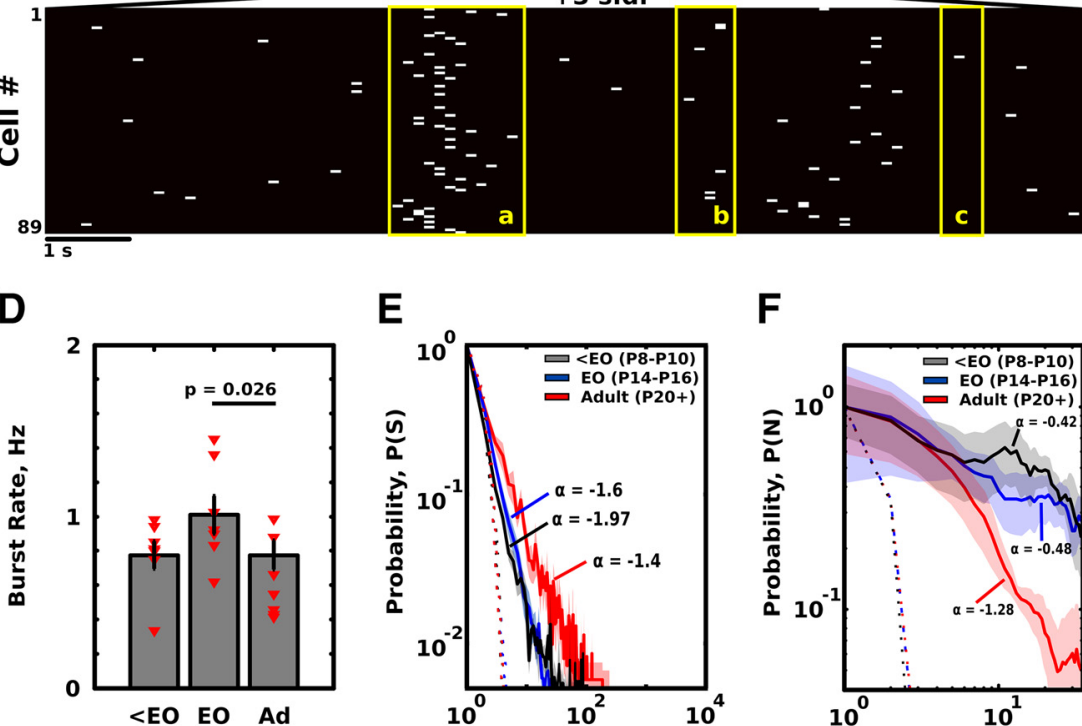

$E$

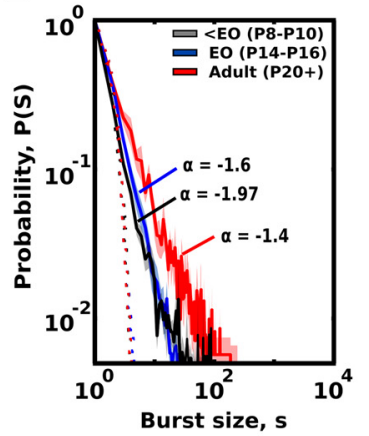

$F$

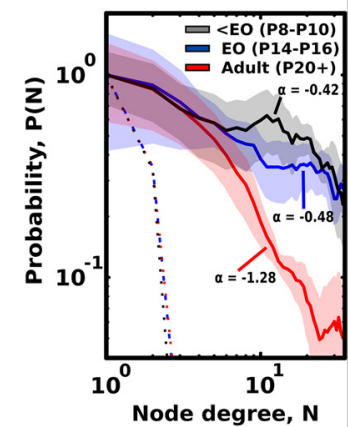

G

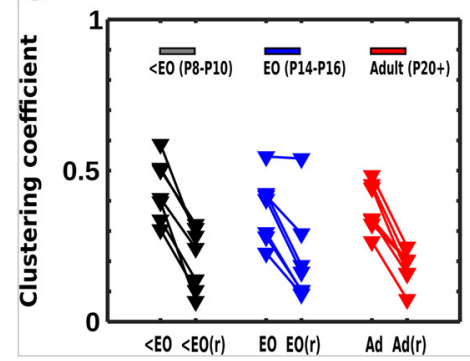

H

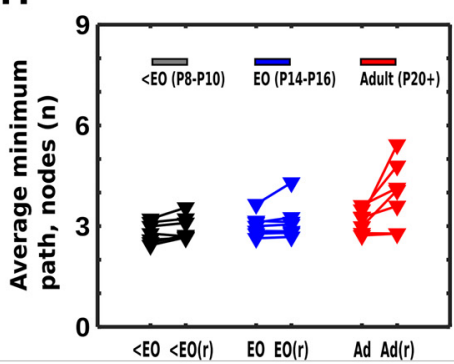

I

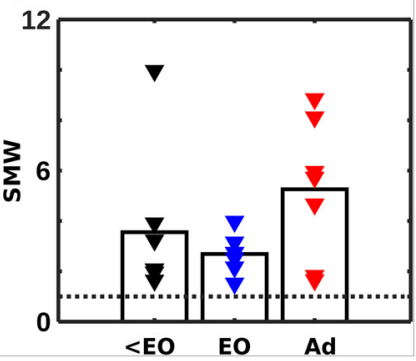

Figure 1. Spontaneous activity organizes as multineuronal population bursts. A, Top, FOV in the primary visual cortex stained with OGB-1 (green cell bodies). Red cells, Td-Tomato labeled Dlx5/6+ interneurons. Light blue, astrocytes (labeled by SR-101). Bottom, Example calcium dF/F signal time courses from cells circled in A. B, Top, Calcium dF/F responses from a population of 89 cells; this excerpt represents $370 \mathrm{~s}$ of recording out of a $30 \mathrm{~min}$ long spontaneous activity movie. White outline, Subregion of the time course expanded in the bottom. Bottom, Restricted portion of the population eventogram (outlined by a white rectangle in top) showing the onset of calcium responses (3 SD above baseline activity) in each individual cell. The ongoing activity contains both solitary cellular bursts (example in Box c) and multineuronal population bursts, involving many cells and extended in time. Multineuronal population bursts (examples are shown in Boxes a and b) are composed of at least two participating cells, whereas solitary cellular bursts (Box c) occur in one cell. The start of a burst is a frame that contains at least one active cell preceded by an empty frame; the end is the frame that contains at least one active cell followed by an empty frame. Yellow Boxes a and b outline two example multineuronal population bursts. Box a, 46 cells, $1.4 \mathrm{~s}$ long population burst. Box b, 7 cells, 0.46 s long burst. Box c, Outline of a solitary single-cell calcium event (solitary burst; size $=1$ cell). Solitary burst events occupy one frame and are preceded and followed by an empty frame. From each FOV we collected between 10 and $40 \mathrm{~min}$ of ongoing activity, containing hundreds of population bursts. Please note that Boxes a-c are just examples and they do not represent all the multineuronal bursts that were identified from this segment of the movie. C, The rates of events in individual cells slightly decrease after eye opening ( $<E 0$, mean \pm SEM, $0.05 \pm$ $0.005 \mathrm{~Hz}, n=7 ; \mathrm{E} 0: 0.036 \pm 0.003 \mathrm{~Hz}, n=7$; Adult: $0.038 \pm 0.002, n=7$; Wilcoxon rank sum test across FOVs, $<\mathrm{E} 0$ vs Adult: $p=0.026, z=2.1, \mathrm{~W}=69.5, r=0.56 ;<\mathrm{E} 0$ vs E0: $p=0.038$, $z=2, W=69, r=0.54$ ). Red triangles, Individual FOV values. $D$, The rate of population bursts (including both multineuronal and solitary bursts) also somewhat decreases after eye opening (EO vs Adult: $p=0.026, z=2.17, \mathrm{~W}=70, r=0.58$, Wilcoxon rank sum test across FOVs; $<\mathrm{E} 0$, mean $\pm \mathrm{SEM}, 0.78 \pm 0.08, n=7 ; \mathrm{E} 0: 1.0 \pm 0.11, n=7 ;$ Adult: $0.62 \pm 0.1, n=7)$. Red triangles, Individual FOV values. $\boldsymbol{E}, \boldsymbol{F}$, Properties of population bursts follow scale-free distributions ( $<\mathrm{E} 0, n=7 ; \mathrm{E} 0, n=7 ; \mathrm{Adult}, n=7)$. $\boldsymbol{E}$, The distribution of the burst sizes obeys a power law $(\alpha=-1.4$ to -1.97 ) at every developmental stage tested ( $<$ E0: P8 - P10; E0: P12-P16; Adult: P35+). Solid lines, Mean across individual FOV distributions; shading, SEM. Dotted lines, Control, distribution of burst sizes after random shuffling of cell event onsets. $F$, The distributions of node degrees, i.e., the number of significant functional links formed by each neuron (see Materials and Methods), are also scale-free at every developmental stage. However, now the power law changes slope (from -0.42 before eye opening to -1.28 in adulthood) reflecting a reduction in the number of functional links with postnatal maturation. Solid lines, Mean across individual FOV distributions; shading, SEM. Dotted lines, Control, distribution of significant links after random shuffling of cell event onsets. G-I, V1 layer 2/3 V1 networks display small-world properties at every developmental stage. G, Clustering coefficients in L2/3 networks are larger than corresponding clustering coefficients in random networks with similar average number of links per node. $<\mathrm{E} 0$, Before eye opening; $\mathrm{E} 0$, around eye opening; Ad, adult. $<\mathrm{EO}(r)$, EO(r), and $\mathrm{Ad}(r)$ represent corresponding clustering coefficient values obtained from random networks with similar average number of connections per cell. These values were conservatively taken to be 3 SD ( $99.7 \%$ cutoff) above the mean of a null distribution of clustering coefficient values obtained by randomly reassigning existing connected pairs across the network nodes 5000 times (see Materials and Methods, Small-world network parameters and analysis). $\boldsymbol{H}$, Average minimal path length stays similar across ages and is slightly reduced compared with random networks with similar total number of nodes and average number of links per individual node. Conventions are similar to G. I, SMW obtained by normalizing the real clustering coefficient and average minimum path length by those of corresponding randomly connected networks (see Materials and Methods) exceeds 1 (gray dotted line) at every age examined. 
A
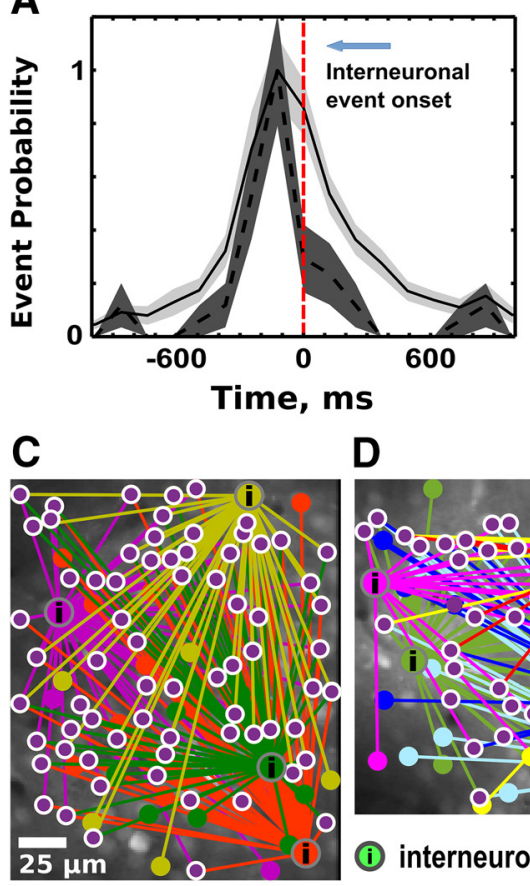

$\mathbf{F}$

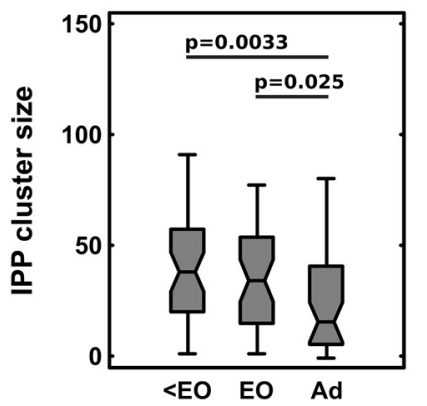

B

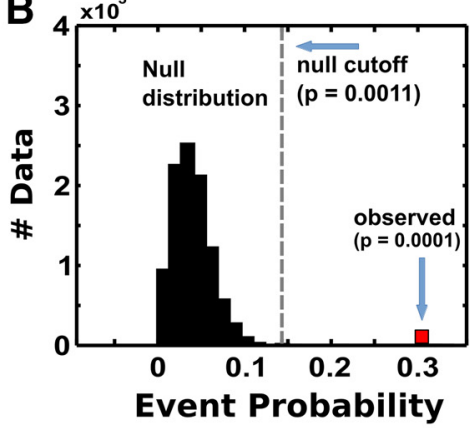

E
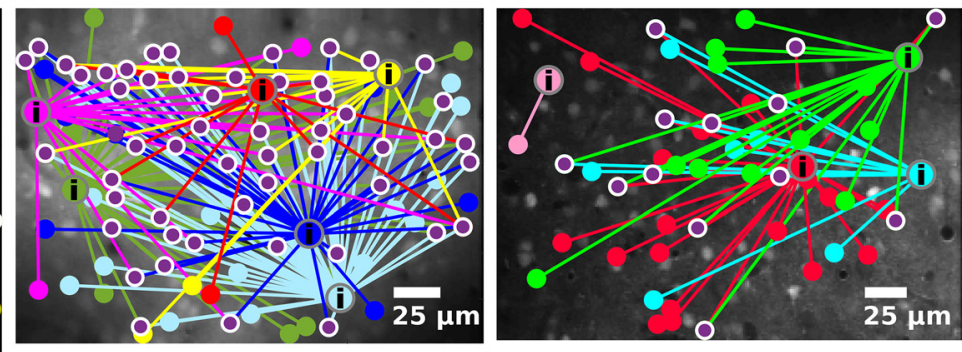

(i) interneurons; $\odot$ pyramidal members;

O shared pyramidal members

G

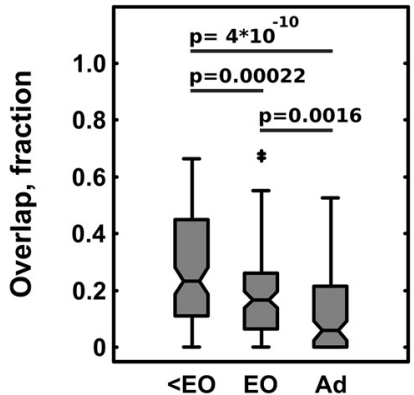

H

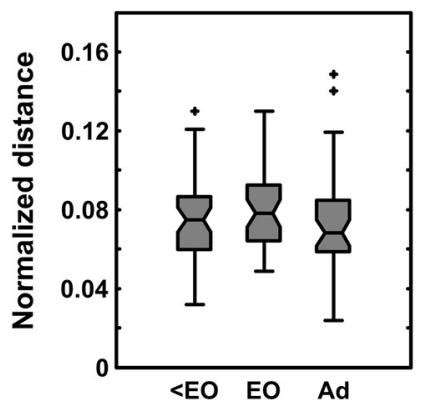

Figure 2. IPP clusters. Identifying PCs with increased probability to generate calcium events before the interneuron's events. $A$, Normalized histogram of $P C$ events occurring -1000 to $1000 \mathrm{~ms}$ around the calcium response onsets of the interneuron. Dotted black line, Example of a single PC (mean over 65 trials). Solid black line, Aggregate responses of all PCs in an FOV whose probability of firing was increased before the interneuron's events. Filled patches, SEM across all trials. B, To assess significance, we first selected out PCs which had larger probability of events in the 600 ms period before the events of the interneuron compared with $600 \mathrm{~ms}$ period after the events of the interneuron. Next, the event trains of these PCs were randomly shuffled cell-by-cell, in circular fashion, to generate the 10000 point null distribution of PC event probability in $600 \mathrm{~ms}$ window after the events of the interneuron. The significance of the difference for each prospective pyramidal partner could then be determined by comparing the PC's event probability before interneuron's event relative to the null distribution (black histogram). The threshold $p$ value was set at 0.05 after correction for multiple comparisons (in the example above this corresponds to initial uncorrected $p$ value of 0.0011 ). Cells with $p$ values below the threshold were accepted as partners, denoting a link between the interneuron and the corresponding $\mathrm{PC}$ (red bar; uncorrected $p$ value is 0.0001 , corresponding to 0.0045 after correction). $\boldsymbol{C}-\boldsymbol{E}$, Examples of IPP clusters at different developmental points. C, P9; D, P15; $\boldsymbol{E}$, P35. IPP-cluster members are color coded. i, Interneurons; filled disks, pyramidal members; circled disks, shared pyramidal members. Scale bars, $25 \mu \mathrm{m}$. $\boldsymbol{F}$, IPP cluster size decreases significantly after eye opening and into adulthood: ( $<\mathrm{E} 0$ : P8 $-\mathrm{P} 10, n=45 ; \mathrm{E} 0$ : P12-P16, $n=41 ; \mathrm{Ad}: \mathrm{P} 35-\mathrm{P} 52, n=38)$. Significance was assessed by Wilcoxon rank sum test across clusters, $<\mathrm{E} 0$ vs adult: $p=0.0033, z=2.4, \mathrm{~W}=2047.5, r=0.26 ; \mathrm{E} 0$ vs adult: $p=0.0249, z=2.2, \mathrm{~W}=1869, r=0.25 ;<\mathrm{E} 0$ vs $\mathrm{E} 0$ (nonsignificant difference): $p=0.4389, z=0.7741, \mathrm{~W}=$ 2047.5, $r=0.08$ ). G, IPP-cluster overlap drops at E0 and further decreases in adult animals. Before E0 mean fraction of overlapping cluster members was $0.28 \pm 0.02$ ( $n=129$ cluster pairs). This reduced to $0.18 \pm 0.015$ ( $n=106$ cluster pairs) at $E 0$ and further to $0.13 \pm 0.016(n=92$ cluster pairs) in adult animals. Significance was determined by Wilcoxon rank sum test across cluster pairs, $<$ E0 vs E0: $p=0.00022, z=3.7, \mathrm{~W}=17136, r=0.24 ;<\mathrm{E} 0$ vs adult: $p=4 \times 10^{-10}, z=6.3, \mathrm{~W}=17242, r=0.42 ; \mathrm{E} 0$ vs adult: $p=0.0016, z=3.2, \mathrm{~W}=11808, r=0.23 . \boldsymbol{H}, \mathrm{The}$ average distance of pyramidal IPP-cluster members from their partner interneuron, normalized for the expected increase in interneuronal distance over postnatal development, remains stable with age (see Materials and Methods). Box plots show the median, with notches encircling the $95 \%$ confidence interval and a box encompassing the interquartile range of the data. Whiskers cover the full range of the data, excluding the outliers. Outliers are marked with crosses.

icant, we used simulated null distribution of Pearson coefficients obtained from circularly shuffled data. The onsets of cellular events were circularly shuffled by applying a variable offset $[1<$ offset $<$ (movie length -1)], where the movie length is the total number of frames in a given movie, for each cell. The offset was selected randomly. Shuffling was repeated 10,000 times to generate the distribution of correlation coefficients that can occur by chance for each cell pair. Each pair's null distribution's maximum value was used as a threshold to determine whether the real correlation coefficient value was significant (e.g., exceeded the threshold). Next, we examined whether the cell pair's real correlation value also was above the median correlation observed in an examined FOV across all cell pairs. If both conditions were satisfied, the cell pair was accepted as having a significant functional link. Links obtained through this procedure were then used to calculate the degree of connectivity across the network nodes (cells).

Small-world networks are defined by a relation between the clustering coefficient and the short average minimum path length. The clustering coefficient $(C)$ for an individual cell (node) is the ratio of the actual links $(s)$ formed between all the linked partners $(k)$ of the cell over the number of all possible links: 


$$
C(\text { node })=2 *(s /(k *(k-1))) .
$$

The global clustering coefficient $\left(C_{g}\right)$ is then determined by averaging the clustering coefficients of all nodes.

Small-world networks also have short path lengths $(L)$, also a common property of random networks. Path length is a measure of the distance between nodes in the network, calculated as the mean of the shortest geodesic distances (number of edges) between all possible node pairs. For nodes $i, j, L$ is determined by:

$$
L=\frac{1}{N(N-1)} \sum_{i \neq j} d_{i j}
$$

where $d_{i j}$ is the shortest geodesic distance between nodes $(i, j)$ and $N$ is the number of the cells in the network. To calculate the small-world factor, we compare the global clustering coefficient and path length of the actual network with the global clustering coefficient $\left(C_{g, r a n}\right)$ and path length $\left(L_{r a n}\right)$ of a random network with the same number of nodes and same mean number of links per cell. Such a network was created by randomly redistributing the existing links in the actual network across the nodes. Then we calculated the small-world factor (SMW), as follows:

$$
S M W=\left(C_{g} / C_{g, r a n}\right) /\left(L / L_{r a n}\right) .
$$

SMW $>1$ is used as a criterion to classify the network as a small-world net (Humphries et al., 2006; Sporns, 2011), because it argues for high average clustering coefficient relative to path length compared with otherwise equivalent randomly connected nets. Approximately $8 \%$ of nodes in the network had limited connectivity: they either were not connected to any other nodes in the network, or were connected to only to a very restricted subset of nodes, which resulted in their individual average path length value to be close to zero. We checked whether their inclusion in the calculation affected the resulting SMWs. To do so, we either imputed the mean path length for them with the mean path length across the connected nodes, or excluded them from the calculation all together. In both cases the SMWs decreased only slightly, with lowest values staying $>1.64$ in all cases.

Identifying interneuron pyramidal partners. To determine whether the activation of PCs and interneurons in the context of multineuronal population bursts was random, or alternatively, groups of specific PCs and interneurons were working in concert, we compared the probability of individual PCs having a calcium event $600 \mathrm{~ms}$ before and $600 \mathrm{~ms}$ after the event in the interneuron.

For each PC, we generated a probability difference measure, by subtracting the probability of PC event $600 \mathrm{~ms}$ before the interneuronal event from the probability of PC event $600 \mathrm{~ms}$ after the interneuronal event, and selected out the cells with positive difference values for further analysis, as these cells potentially had higher probability to be activated shortly before interneuronal events. The probability difference measure was calculated over (13-92) instances of interneuronal activations. If the interneuron had $<12$ in-burst calcium events, we did not consider it for further analysis.

We then generated 10,000 instances of surrogate datasets by temporally shuffling the event onsets in these potential PC partners, and leaving the interneuronal event onsets intact. For each shuffled dataset and each potential PC partner, a surrogate "chance" probability of a PC to be activated after the interneuronal event was generated (10,000 points). Next, for each tested PC, the real probability to have an event before the interneuronal event was compared against each chance probability point in this null distribution, and the fraction of instances when the chance probability was equal to or exceeded the real probability was used as a $p$ value for each interneuron-PC pair. Individual cells' $p$ values were then adjusted for multiple comparisons (by multiplying them by the number of tested PC partners), and PCs with adjusted $p$ values $<0.05$ were selected as the partners of the interneuron. $95 \%$ of adjusted $p$ values were $<0.044$, with median value at 0.016 .

The groups of PCs whose probability of firing was significantly increased before the event in the interneuron, were then called IPPs and assigned to a single cluster (IPP cluster).
The $600 \mathrm{~ms}$ integration window was selected based on two considerations. First is the dynamics of ongoing excitatory and inhibitory population inputs into the layer $2 / 3$ PCs observed in intracellular recordings data (Douglas and Martin, 1991). In individual layer 2/3 neurons, ongoing network activity resulting from visual stimulation causes fast population EPSP, followed by prolonged population IPSP, which lasts for 250-350 ms after the population EPSP onset (Douglas and Martin, 1991). Second is the relative response latency of oriented interneurons and PCs to preferred stimulus in our data (15 interneurons and 431 PCs, collected from 4 FOVs of 4 adult animals). Under preferred visual stimulus there was $140 \mathrm{~ms}$ median lag between pyramidal and interneuronal population response onsets (calculated across all trials and all cells in the given FOV). However, if we only considered PC trials with shorter latencies relative to the interneuronal trials, the median lag increased to 600 $\mathrm{ms}$, which was subsequently chosen as the window for determining the presence of a functional link between PC and interneuron.

Global functional connectivity of the PCs and group correlation strength of the IPP clusters. We next checked whether event trains of individual IPP cluster members had different group correlation strength between themselves compared with other pyramidal neurons in the FOV that did not belong to the cluster. For this, we first determined which PC pairs in the FOV had significant cross-correlations within the test window used to determine the membership of PCs in IPP clusters. The cross-correlation analysis was done on the eventogram binary data within a -600 to 600 ms window, where $t=0$ corresponds to the PC's event. Pairwise linear Pearson coefficient was computed for every PC pair. To determine whether the coefficients were significant, we generated 10,000-iteration null distributions of values, by circularly shuffling the event onsets in every cell. The displacement values for each cell were selected at random. Using this 10,000-point null distribution, we looked whether the actually observed cross-correlation for a specific pair was outside the $99.7 \%$ values of the null distribution to accept the value as significant and not occurring by chance. Pairs with a significant value were counted as a functional "link". The nonsignificant coefficients were set to zero. We then used the resulting matrix of significant functional links ("significance matrix") to explore the group correlation strength within the IPP clusters.

For this, we first removed periods of interneuron activation $[(-600$ $\mathrm{ms}, 600 \mathrm{~ms}$ ) periods around each interneuronal event] from the FOV's eventogram. Next, the pairwise cross-correlation was computed between every PC pair in the remaining portion of the eventogram, using a window of -600 to $600 \mathrm{~ms}$. We then used significance matrix to determine whether the link between a particular pair of cells was significant. Nonsignificant links' correlation values were put to zero. Then, mean crosscorrelation coefficient was measured for PC pairs of the same IPP cluster. To determine whether this mean peak cross-correlation was significantly different from control, we generated 10,000 surrogate cell groups, size matched to the tested IPP cluster, by randomly selecting PCs out of the FOV without replacement. We used the following criteria to select the member cells for these control groups (Fig. 3):

(1) Their pairwise distances to the partner interneuron were restricted to match the pairwise distance range of the tested IPP cluster members to the partner interneuron. For this, we averaged distances from the interneuron to each partner PC in the cluster. The distance envelope was set to mean $\pm 3 \mathrm{SD}$ from the interneuron. (2) The cells did not belong to an IPP cluster. (3) We then calculated the mean pairwise crosscorrelation coefficient of the eventogram between the members of the surrogate group and the members of the tested IPP cluster. This was repeated 10,000 times to arrive at the null distribution of mean correlation strength of randomly selected cell groups in the given FOV. Using this null distribution, we determined whether the IPP cluster's mean pairwise cross-correlation peak value was outside of the $p=0.997$ interval (3 SD from the mean).

Global functional connectivity of the PCs during multineuronal bursts. We next used the significance matrix to explore the refinement of functional connectivity in the course of early development. We set all nonzero values to 1 , denoting a presence of a functional link between the cells, whereas non-linked cells were assigned a 0 . We next used the resulting matrix to determine the amount of links made by pyramidal neurons 
A

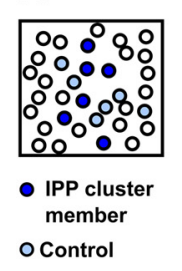

B

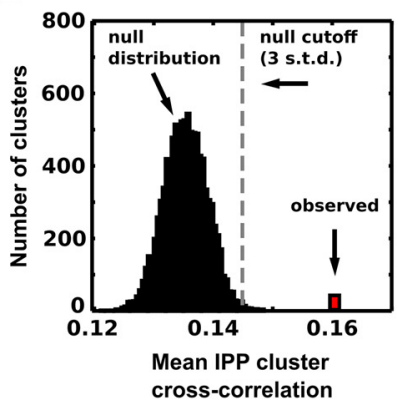

C

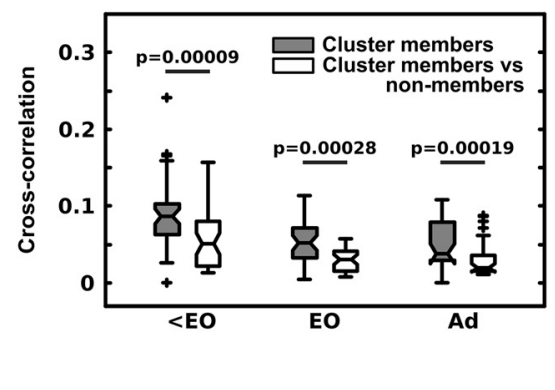

D

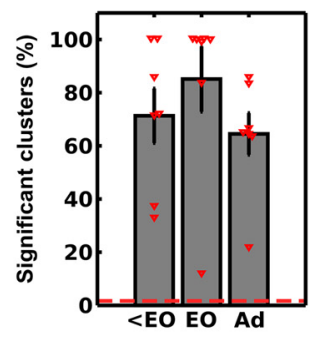

Figure 3. IPP-cluster members show higher pairwise cross-correlation strength. $\boldsymbol{A}$, Dark blue, IPP cluster members. Cyan, Size- and distance-matched control sample of randomly selected PCS from the same FOV. This control cluster has to satisfy the following requirements: (1) its size corresponds to the original cluster's size, and (2) its members have to be on average at the same distance from the original IPP cluster's partner interneuron as the IPP cluster's pyramidal members (see Materials and Methods). B, Null distribution (black histogram) of mean pairwise Pearson crosscorrelation values ( $600 \mathrm{~ms}$ window) computed between PCs, one of which belongs to the IPP cluster, the other to a control cluster (see Materials and Methods). Ten thousand control clusters were randomly selected per IPP cluster within the FOV. Significance required that the mean pairwise correlation coefficient of the IPP cluster (red) be $>99.7 \%$ of the null distribution values. $\boldsymbol{C}$, Pairwise cross-correlation strength between IPP-cluster pyramidal partner members that belong to the same cluster (gray bars) versus pyramidal nonmembers, after controlling for pairwise distance (see Materials and Methods). Pairwise correlation strength decreases over time in agreement with the decorrelation that occurs in early postnatal development (Golshani et al., 2009; Rochefort et al., 2009): before eye opening mean in-cluster correlation was $0.087 \pm 0.006$ (median, 0.086 ); significantly dropping to $0.053 \pm 0.005$ (median, 0.052 ) after eye-0pening and stabilizing at $0.05 \pm$ 0.005 (median, 0.037) in adult animals $[<\mathrm{E} 0, n=44 ; \mathrm{E} 0, n=40 ;$ adult, $n=36$ clusters from 7 FOVs; Wilcoxon rank sum test results (over clusters), $<\mathrm{E} 0$ vs $\mathrm{E} 0: p=0.00014, z=3.8, \mathrm{~W}=2296$, $r=0.41 ;<$ E0 vs adult: $p=2.5 \times 10^{-5}, z=4.2, \mathrm{~W}=2218, r=0.47$; EO vs adult (nonsignificant difference): $\left.p=0.3682, z=0.9, \mathrm{~W}=1627, r=0.103\right]$. At all ages, average pairwise cross-correlation strength was significantly higher within versus across different IPP clusters (significance determined by Wilcoxon rank sum test, $<$ E0: within clusters, $0.087 \pm 0.006$, across clusters $0.057 \pm 0.006, n=44$ clusters, $p=0.00009, z=4, \mathrm{~W}=2424, r=0.6 ; \mathrm{E} 0$ : within clusters, $0.053 \pm 0.005$, across clusters, $0.03 \pm 0.0025, n=40$ clusters, $p=0.00028, z=3.6, \mathrm{~W}=$ $1998, r=0.57$; adult: within clusters, $0.05 \pm 0.005$, across clusters, $0.03 \pm 0.004, n=36$ clusters, $p=0.00019, z=3.73, W=1646, r=0.62$ ). $\boldsymbol{D}$, Percentage of IPP clusters whose members have significantly higher mean cross-correlation strength than control, using the criterion described in $\boldsymbol{B}$. Chance level is $1 \%$ (red dotted line). The percentage of IPP clusters with significantly higher mean pairwise cross-correlation strength than control is already substantial before eye opening ( $\sim 74 \%$ for $<$ E0: $n=44$ clusters from 7 FOVs, mean \pm SEM: $0.71 \pm 0.01$ ) and remains high (65-85\%) after eye opening (E0: $n=40$ clusters from 7 FOVs, mean \pm SEM: $0.85 \pm 0.12$; Adult: $n=36$ clusters from 7 FOVs, mean \pm SEM: $0.65 \pm 0.08$ ). Clusters consisting of the interneuron and single connected PC were excluded from analysis. Red triangles, Individual FOV values. Box plots show the median, with notches encircling the $95 \%$ confidence interval and a box encompassing the interquartile range of the data. Whiskers cover the full range of the data, excluding the outliers. Outliers are marked with crosses.

that participated in a singular IPP cluster ("exclusive” cell) versus the amount of links made by pyramidal neurons participating in more than one IPP cluster ("shared" cell).

Statistical tests. We used nonparametric tests as described in the text and the figures to compare cluster sizes, member overlap, and functional connectivity within and between clusters in different age groups. We also used nonparametric tests to assess alignment of visual tuning between cluster members. Significance was defined as $p<0.05$ (adjusted to account for multiple tests by Benjamini-Hochberg procedure with false discovery rate controlled at 0.05 , when more than one comparison was made) and corresponding $Z$-score outside of the $-1.96,1.96$ range. Unpaired data were compared using two-tailed Wilcoxon rank sum test. Two-tailed Wilcoxon signed rank test was applied in cases when the group data were paired or single-sampled (ratio data compared against $1)$. For both tests the following are reported: sum of ranks (W), $Z$-score $(z)$, effect size $(r)$, and exact $p$ value of the test. Nonsignificant results are also reported and marked as nonsignificant. The tests were performed using standard MATLAB (MathWorks) functions. Box plots show the median, with notches encircling the $95 \%$ confidence interval and a box encompassing the interquartile range of the data. Whiskers cover the full range of the data, excluding the outliers. Outliers are marked with crosses. Error bar plots show mean \pm SEM (standard error of the mean) and locations of individual data points. The mean \pm SEM and median values of the data are given in the text and figure legends.

Selectivity for the direction(s) of stimulus motion. The dF/F of each evoked calcium data was de-convolved using the algorithm of Vogelstein et al. (2010). Resulting output data were used to construct the cell's tuning curve for drifting gratings. We evaluated the selectivity of each cell for the direction of motion of drifting gratings using the direction selectivity index (DSI):

$$
D S I=\left(D_{\text {preferred }}-D_{\text {opposite }}\right) /\left(D_{\text {preferred }}+D_{\text {opposite }}\right),
$$

where $D_{\text {preferred }}$ is the response for motion to the cell's preferred direction, and $D_{\text {opposite }}$ to the opposite direction. Highly selective cells have DSI near 1, whereas a 3:1 response difference would result in a DSI of 0.5.
The cells that generate bi-peaked tuning curves for the drifting grating are characterized by orientation-selectivity index, as those peaks are typically opposite or near-opposite each other:

$$
\text { OSI }=\left(O_{\text {preferred }}-O_{\text {orth }}\right) /\left(O_{\text {preferred }}+O_{\text {orth }}\right),
$$

where $O_{\text {preferred }}$ is the direction axis (orientation) along which peak responses occurs, while $O_{\text {orth }}$ is the direction axis (orientation) orthogonal to the peak response axis. We considered the cell to be tuned for direction and/or orientation in case that either of the metrics exceeded 0.45 . Of 23 visually-responsive interneurons in adult dataset (collected from 4 FOVs), 15 were tuned for the direction and/or orientation of the moving gratings (DSI (direction selectivity index) or OSI (orientation selectivity index) equal or $>0.45$ ). We then examined the tuning curve of each interneuron and excluded three cells with exceptionally broad tuning, that had $>3$ significant peaks in their tuning curve (for this we determined the amplitude of the largest peak, and accepted any other peaks that were more than half of that amplitude as significant). This left us with 12 interneurons for the grating condition.

For all examined tuned interneurons we first identified the dominant peak in their tuning curve. Most cells also had additional smaller peaks in their tuning curves, typically located opposite of the dominant peak, as most of the oriented neurons in V1 show a degree of orientation preference. We thus looked whether the interneuron had additional peaks located in the $120^{\circ}$ window centered on the direction opposite to the dominant peak. The minor peak was considered significant if the amplitude of the local maximum was significantly $>0$. We considered both peaks as "preferred direction" when evaluating the alignment of tuning between the tuning curves of the interneuron and its partner PC (see Fig. 5).

We then examined the alignment between the tuning curves of the interneurons and the PCs found in their corresponding clusters. First, we examined the population tuning curves of pyramidal cluster members versus interneuron (Fig. 5C). Because the communal tuning curve appears to be dominated by oriented pyramidal members, we proceeded to describe the relationship between their tuning and the tuning of the 
interneuron. For each cluster, we selected out cells that had sufficient direction or orientation tuning. First, we removed cells whose OSI or DSI was $<0.45$. We examined the tuning curves of the remaining cells and removed cells with noisy peak responses and cells whose tuning curves had more than two significant peaks (a significant peak reached $2 / 3$ of the amplitude of the dominant peak). For the remaining cluster members, we determined the smallest offset of the preferred direction of each pyramidal neuron from the preferred directions of the partner interneuron.

\section{Results}

Scale-free population burst-events in layer 2/3 of mouse area V1 reveal small-world network architecture

We imaged spontaneous ongoing activity in the developing postnatal and juvenile-adult visual cortex of mice using two-photon calcium imaging. OGB-1 dye was used to densely label neocortical neurons at early postnatal ages. One to 4 movies were acquired per FOV at 3.23-11 Hz under light (0.6\%) isoflurane anesthesia (10-40 min in total). FOVs contained on average 148 (78-292) neurons whose calcium signals were acquired simultaneously (Fig. 1A,B). The onset of individual OBG calcium events was identified by thresholding the $\mathrm{dF} / \mathrm{F}$ trace at $+3 \mathrm{SD}$ from the level of noise in each cell (Fig. 1B). The threshold selected ensured that identified calcium events reflect burst spiking events with high likelihood: $77 \%$ of spike burst onsets (doublets and multiplets) were detected reliably (see Materials and Methods, Population bursts). All identified neurons generated spontaneous calcium events. For each neuron, we assigned the number 1 to frames containing a calcium event onset, whereas frames with no event onsets were assigned the number 0 . This yields a binary "eventogram" of calcium event onsets across the whole imaging period. We then grouped cellular events into spatiotemporal bursts (Fig. $1 B$, bottom shows some examples). The beginning of a burst was defined as a frame that contained at least one cell calcium event onset following an event-free epoch. The burst continued as long as consecutive frames contained at least one calcium event onset in one of the FOV cells and ended on encountering an event-free frame. Two types of burst events are observed in this case: multineuronal bursts (involving more than one cell, size $>1$ ) and solitary events (single-cell bursts; involving only 1 cell in the plane of imaging). We detected on average 1200 spontaneous burst-events per FOV over 24 min of recording, of which 55\% were multineuronal bursts ( size $>1$; individual FOV burst-event rate $0.32-1.35 \mathrm{~Hz}$; Fig. 1D). Burst-event rate decreased slightly during postnatal development, following the event rate decrease seen in individual cells (Fig. 1C,D). At every postnatal age examined the distribution of burst-event sizes was scale-free and conformed to a power law (Fig. $1 E$ ). Scale invariance suggests that firing events happen at all spatial scales up to the size of the studied FOV. The scaling factor $(\alpha)$, i.e., the slope of the linear $\log -\log$ plot of burst-event probability versus size, characterizes the relative probability of occurrence of burst-events of different size. Scaling factors for burst-event sizes remain close $(\alpha=-1.4$ to -1.97 ) throughout all ages examined (Fig. $1 E$ ), from before eye opening (P8-P10) to juvenile adulthood $(\mathrm{P} 35+)$. The fact that burst-event sizes obey a power law implies that individual neuronal events are functionally coupled (Eurich et al., 2002; Beggs and Plenz, 2003) throughout postnatal development

To further analyze the structure of synchronous events, we assigned a link between pairs of cells whose Pearson correlation coefficients reached a desired level of significance $(p<0.0001$; see Materials and Methods, Small-world network parameters and analysis). The number of links emanating from each cell measures the cell's "degree of connectivity", i.e., the number of neighboring units with above chance probability to fire in synchrony with that cell. The degree of functional connectivity across L2/3 cells also obeyed a power law (Fig. $1 F$ ), suggesting an underlying hierarchical small-world network architecture (Sporns, 2011). This architecture features "cliques" of functionally interconnected cells forming sparse links with each other via relatively rare clique cross-connecting hub nodes (Bassett and Bullmore, 2006; Sporns, 2011). Hierarchical small-world nets are characterized by large average clustering coefficient to average minimal path length ratios compared with randomly connected nets (see Materials and Methods), i.e., by a SMW > 1 (Sporns, 2011; Fig. $1 G-I$ ). V1 layer $2 / 3$ has SMW $\gg 1$ (range $1.52-9.96$ ) at every postnatal age examined (Fig. 1I). Interestingly, the magnitude of the scaling factor for the degree of connectivity increased dramatically with postnatal maturation (from -0.42 to -1.28 ; Fig. $1 F$ ), suggesting gradual functional link elimination and subsequent decrease in the size of highly connected cliques over time after eye opening (Fig. $1 F$ ). This is in line with spontaneous activity decorrelation during the first weeks of postnatal development (Golshani et al., 2009; Rochefort et al., 2009; Fig. 3C).

Small-world cliques of neurons in the adult brain have been reported in the literature (Humphries et al., 2006; Sporns and Honey, 2006; Meunier et al., 2010) and are proposed to play an important role in cortical computations (Lago-Fernández et al., 2000). Note, however, that not every cortical network conforms to small-world structure; modularity may also coexist with dense connectivity, as in case of long-range anatomical connectivity in macaque brain (Markov et al., 2013). We aimed to identify and characterize putative small-world cliques in L2/3 of mouse area V1 and investigate how they evolve during development. Our approach is guided by the intuition that cliques of functionally similar PCs whose synchronous firing represents a "temporally coherent computational unit" (or feature) ought to be inhibited together. Therefore, clique members are expected to form functional connections not just with each other, but also with the distinct set of local interneurons.

\section{Subnetworks of pyramidal neurons temporally linked to specific interneurons}

To identify candidate interneuron-linked small-world groups we searched for cells whose calcium events had a consistent temporal relationship to events recorded from local inhibitory interneurons. We used mice expressing Td-Tomato in Dlx-5/6-positive interneurons, which labels $\sim 98 \%$ of layer $2 / 3$ interneurons, including all major subtypes of neocortical interneurons $(\mathrm{PV}+$, SOM+, VIP+ and others; Stühmer et al., 2002; Monory et al., 2006; Madisen et al., 2010; Rudy et al., 2011). For each interneuron, we identified the PCs that generated events with higher probability within the $600 \mathrm{~ms}$ window immediately preceding interneuronal firing events (Fig. 2A). This relatively large window reflects the build-up of recurrent network activity rather than purely monosynaptic connections, which are faster (see Materials and Methods, Identifying interneuron pyramidal partners). Significance was estimated by random circular shuffling of calcium response onsets to build a null distribution of $\mathrm{PC}$ event probability relative to interneuronal events [Fig. $2 B ; p=0.0001(0.0045$ after correcting for multiple comparisons), and less than cutoff $p$ value of $p=0.0011$ (which corresponds to 0.05 after correction for multiple comparisons)]. These PCs have the potential of activating the interneuron and then being themselves inhibited by the interneuron in the context of recurrent network activity. We refer to such cells as "partners" of that interneuron. An interneuron with its associated pyramidal partners constitute a clique we call "interneuron pyramidal partner cluster" (IPP cluster; Fig. 
$2 C-E)$. Nearly all (97\%) interneurons had pyramidal partners inside the FOV. Interestingly, the average distance of IPP cluster pyramidal members from the partner interneuron remained stable across the ages examined (Fig. $2 \mathrm{H}$ ) when normalized appropriately by the increase in inter-neuronal distance (expected during the course of postnatal development) and FOV size (maximal distance between cells; Fig. $2 H$ ). Note that IPP cluster identification depends on functional connectivity between interneurons and PCs without implying anything about the underlying anatomical substrate.

\section{Evolution of IPP clusters during postnatal development}

The number of neurons per IPP cluster as well as average overlap between IPP clusters decreased significantly during postnatal development (Fig. 2F,G). Specifically, before eye opening (P8P10) mean IPP cluster size was $38.6 \pm 3.5$ pyramidal neurons $(n=45$ clusters, mean \pm SEM), around eye opening (P12-P16) $34.4 \pm 3.4(n=41)$, whereas in juvenile adulthood (P35-P52) it dropped further to $24.2 \pm 3.5$ cells $(n=38$; Fig. $2 F)$. Correspondingly, fraction of overlapping members between the clusters was $0.28 \pm 0.02$ (mean \pm SEM, $n=129$ cluster pairs) before eye-opening, $0.18 \pm 0.015$ around eye opening $(n=106$ cluster pairs), and $0.13 \pm 0.015$ in juvenile adulthood ( $n=92$ cluster pairs; Fig. $2 G$ ). Note that there was no systematic difference in the fraction of active cells per FOV across different groups and IPP cluster size/overlap did not correlate with the number of active neurons in the FOV.

To measure the strength of pairwise functional connectivity within a cluster we used linear Pearson cross-correlation function over a $\pm 600 \mathrm{~ms}$ window after removing all [ $-600 \mathrm{~ms}, 600 \mathrm{~ms}$ ] periods centered on interneuronal responses to exclude periods in which interneurons can directly influence their pyramidal partners (or vice versa; see Materials and Methods, Global functional connectivity of the PCs). Pairwise functional connectivity strength was greater between pyramidal IPP cluster members versus IPP cluster members and appropriately distance matched nonmembers from the same FOV (Fig. 3C; see Materials and Methods). The difference in mean pairwise functional connectivity strength was significant already before eye-opening and remained significant through adulthood (Fig. 3C). We computed the mean pairwise connectivity strength across all pyramidal members of an IPP cluster and compared it to that derived from a null distribution generated by creating 10000 surrogate sizematched and distance-matched PC groups randomly drawn cell by cell from the FOV without replacement (see Materials and Methods; Fig. $3 A, B$ ). IPP clusters with mean pairwise functional connectivity strength $>99.7 \%$ of the null distribution's values were conservatively deemed to have mean pairwise functional connectivity strength higher than chance. Remarkably, the majority of IPP clusters exceeded this threshold; regardless of postnatal age the fraction of such clusters exceeded $60 \%$ (Fig. 3D). Note that at the threshold chosen only $1 \%$ of IPP clusters were expected to reach significance by chance, confirming that IPP clusters represent well defined cliques of relatively densely interconnected cells.

"Cluster-exclusive" PCs, i.e., cells that participate exclusively in a single IPP cluster form fewer functional links than shared PCs (cells that participate in more than one IPP cluster) at all ages examined (Fig. $4 A, B$ ). However, both cluster-exclusive PCs and shared PCs retain fewer functional links over time and the number of functional links per pyramidal IPP-cluster member decreases over postnatal development (Fig. 4A,B). The fraction of cluster-exclusive PCs greatly increases after eye opening (Fig.
$4 C$ ), whereas the fraction of shared PCs correspondingly decreases. As a result, by juvenile adulthood a PC member of an IPP cluster is on average functionally linked to $\sim 2(2.1 \pm 0.13$, mean \pm SEM, $n=38$ clusters) interneurons, down from $\sim 4$ (3.6 \pm 0.2 , mean \pm SEM, $n=45$ clusters) before eye opening (Fig. $4 D$ ). These processes reflect the refinement of small-world network structure, with IPP clusters becoming increasingly segregated, reflecting perhaps an increase in functional specialization (Gao et al., 2010).

Our findings argue strongly that IPP clusters represent neuronal cliques existing as early as P8, whose structure undergoes marked reorganization during early postnatal development. Specifically, IPP clusters decrease in size and become more disjoint as functional connections between their pyramidal members get pruned over time. Notably, the average number of IPP clusters linked per shared PC decreases over time, as the network acquires incrementally stronger SMW structure. So far, all analysis was performed on epochs of spontaneous activity. The question then arises whether IPP-cluster members share functional properties, and whether shared nodes cross-link IPP clusters with similar functional properties.

\section{IPP-cluster members share physiological properties}

We found that pyramidal members of IPP clusters share featureselectivity with each other and with their partner interneurons. This analysis was performed in four juvenile adult animals (P42P52, 4 FOVs), in which direction and orientation selectivity are mature (Rochefort et al., 2011). A significant proportion of area V1 visually responsive neurons are either direction or orientationselective (Rochefort et al., 2011). If IPP clusters represent functionally significant modules of computation, it is expected that their members will share selectivity for specific features of the visual stimulus, such as direction or orientation. We focused our analysis on interneurons displaying selectivity for orientation and/or direction of motion of the drifting grating, because $52 \%$ $(12 / 23)$ of visually responsive interneurons recorded in adult animals showed direction- and/or orientation-selectivity when tested with drifting gratings. We discarded nonselective interneurons and their clusters from this analysis, focusing only on selective interneurons. This procedure likely discriminates in favor of VIP-negative and PV-negative interneurons, such as SOM+ cells, which are more likely to be tuned for orientation and direction than PV+ and VIP+ cells (Kerlin et al., 2010; Ma et al., 2010). PV + and VIP + cells are typically broadly tuned and not selective for these features (Kerlin et al., 2010; Ma et al., 2010; Mesik et al., 2015, but see Runyan et al., 2010 for examples of oriented PV+ cells). Figure 5, $A$ and $B$, illustrates an example of three direction tuned pyramidal IPP-cluster members linked to their tuned interneuron partner. Note that the peaks of the tuning functions align across the PCs and the interneuron (Fig. 5C). Figure 5, $D$ and $E$, summarizes population data across all IPP clusters derived from oriented interneurons tested with moving gratings: $34 \%$ of pyramidal members share direction preference with their partner interneuron, $75 \%$ are within $\pm 45^{\circ}$, and only $14 \%$ have preference orthogonal (at least $90^{\circ}$ difference) to that of their partner interneuron (Fig. $5 D$ ). Figure $5 E$ shows the comparison between cluster members versus nonmembers in terms of direction preference alignment with cluster's interneuron: $\sim 75 \%$ $[0.754 \pm 0.052$ (fraction) mean \pm SEM, $n=12$ clusters $]$ of oriented cluster members have preferred direction within $\pm 45^{\circ}$ of interneuron's preferred direction, compared with $\sim 59 \%$ of oriented non-members [0.59 \pm 0.042 (fraction), mean \pm SEM, $n=$ 
A

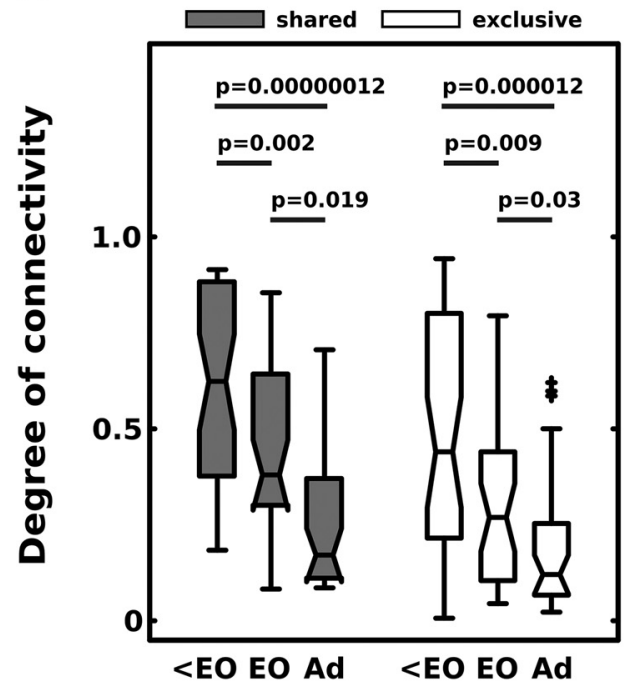

C

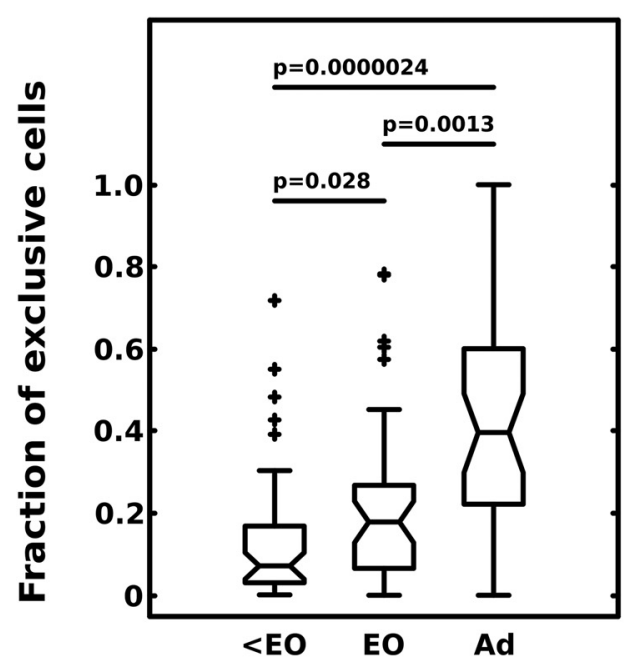

B

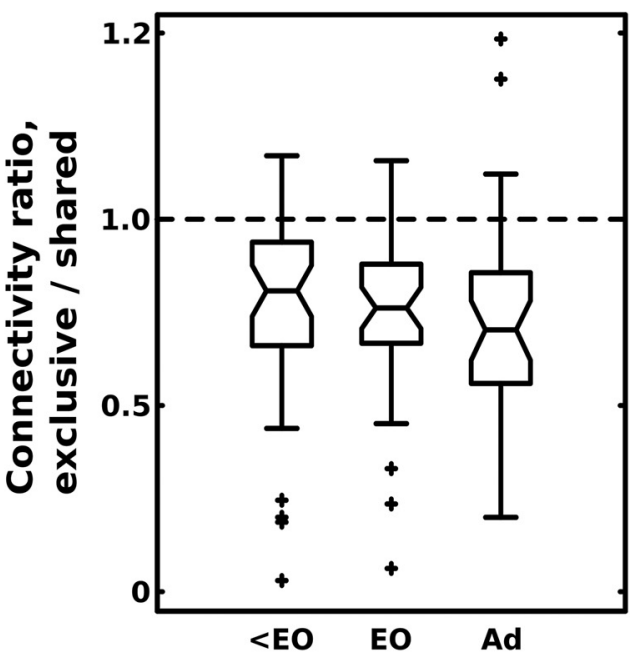

D

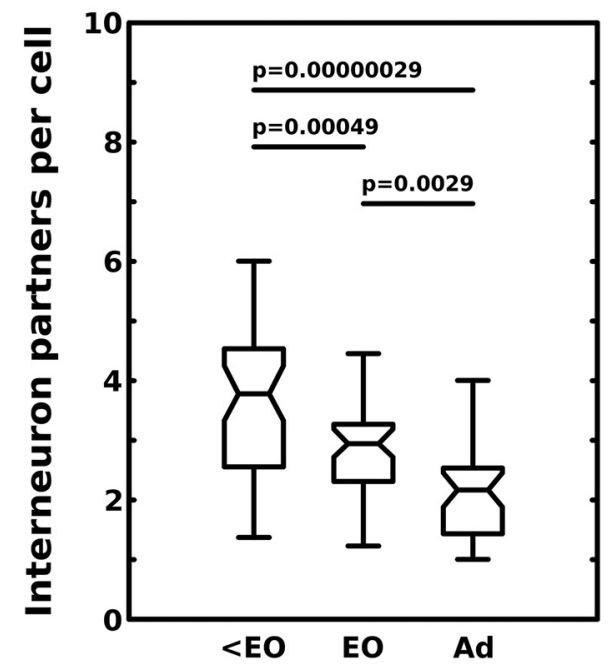

Figure 4. Refinement of IPP-cluster functional connections during development. $\boldsymbol{A}$, The degree of functional connectivity (number of functional links per PC, expressed as a fraction of total number of P(s in the FOV) decreases over the course of early development. On average the mean degree of connectivity for P(s participating in more than one IPP cluster (shared cells; gray bars) drops from $0.61 \pm 0.04$ (median, 0.62 ) before eye opening ( $<$ E0, $n=42$ clusters from 7 FOVs) to $0.41 \pm 0.04$ (median, 0.38 ) around eye opening (E0, $n=35$ clusters from 7 FOVs) to $0.27 \pm 0.04$ (median, 0.17 ) in adulthood (P35,$+ n=33$ clusters from $7 \mathrm{FOVS}$ ). Corresponding numbers for cells participating exclusively in one IPP cluster (exclusive; white bars) are $0.49 \pm 0.05$ (median, 0.44 ) to $0.3 \pm 0.035$ (median, 0.27 ) to $0.205 \pm 0.03$ (median, 0.12 ), respectively. Significance is assessed by Wilcoxon rank sum test. Shared cells, $<E 0$ vs E0: $p=0.0019, z=3.1, W=1813, r=0.35$; $<\mathrm{E} 0$ vs adult: $p=1.2 \times 10^{-7}, z=5.3, \mathrm{~W}=1958, r=0.61 ; \mathrm{E} 0$ vs adult: $p=0.0185, z=2.35, \mathrm{~W}=1400, r=0.29$. Exclusive cells, $<\mathrm{E} 0$ vs $\mathrm{E} 0: p=0.0088, z=2.62, \mathrm{~W}=1767, r=0.3$; $<$ EO vs adult: $p=1.23 \times 10^{-5}, z=4.4, W=1875, r=0.51 ; \mathrm{E} 0$ vs adult: $p=0.03, z=2.2, \mathrm{~W}=1384.5, r=0.27$. $B$, Even though both exclusive and shared cells lose significant number of connections after eye opening and with further development (Fig. $4 A$ ), the degree ratio between exclusive and shared cells belonging to a given IPP cluster is significantly shifted in favor of shared cells for every age group [one-sample signed rank test (against ratio $=1$ ), $<\mathrm{E} 0$ : mean shared/exclusive connectivity ratio $0.76 \pm 0.04$ (median, 0.81 ), $p=6.1 \times 10^{-7} ; z=5, \mathrm{~W}=781, r=0.77$; E0: mean $0.74 \pm 0.04$ (median, 0.76), $p=1.4 \times 10^{-6}, z=4.8, \mathrm{~W}=609, r=0.81$; adult: mean $0.72 \pm 0.05$ (median, 0.7 ), $p=4.1 \times 10^{-5}, z=4.1, \mathrm{~W}=510, r=0.71$ ]. Dotted line $(1$ ) corresponds to the case when there is no difference in degree of connectivity between shared and exclusive cells ( $<$ E0: $n=42$ clusters from 7 FOVs; E0: $n=35$ clusters from 7 FOVs; adult: $n=33$ clusters from 7 FOVs). Note that only clusters containing both exclusive and shared members were used in the analysis for $\boldsymbol{A}$ and $\boldsymbol{B}$. $\boldsymbol{C}$, The mean fraction of PCS that participate exclusively in one IPP cluster dramatically increases after eye opening and with further development, from $0.13 \pm 0.02$ (median, $0.07 ;<\mathrm{E} 0: n=45$ clusters, $7 \mathrm{FOVs}$ ) to $0.22 \pm 0.03$ (median, $0.18 ; \mathrm{E} 0: n=41$ clusters, 7 FOVs) to $0.41 \pm 0.045$ (median, 0.39; adult: $n=38$ clusters, 7 FOVs). Significance was assessed with Wilcoxon rank sum test (across clusters, E0 vs $<\mathrm{E} 0: p=0.028, z=2.2, r=0.24, \mathrm{~W}=2038$; adult vs $<\mathrm{E} 0: p=2.4 \times 10^{-6}, z=4.7, \mathrm{~W}=2112.5, r=0.52 ;$ adult vs $\left.\mathrm{E} 0: p=0.013, z=3.2, \mathrm{~W}=1848.5, r=0.36\right)$. $\boldsymbol{D}$, The number of interneuron partners per $\mathrm{P}($ decreases on average during developmental refinement from pre-eye opening $3.6 \pm 0.2$ (median, 3.8; $<$ E0: $n=45$ clusters, 7 FOVs), to $2.7 \pm 0.1$ (median, 2.9 ) at eye opening (E0: $n=41$ clusters, 7 F0Vs) to $2.1 \pm 0.1$ (median, 2.16) interneurons/PC in adult animals (adult: $n=38$ clusters, 7 FOVs). Significance of this decrease was confirmed by Wilcoxon rank sum test across clusters; $<\mathrm{E} 0$ vs E0: $p=0.0029, z=$ $3, \mathrm{~W}=2302, r=0.32 ;<$ E0 vs adult: $p=2.6 \times 10^{-7}, z=5.2, \mathrm{~W}=2454, r=0.57 ; \mathrm{E} 0$ vs adult: $p=0.0005, z=3.5, \mathrm{~W}=1955.5, r=0.4$. Box plots show the median, with notches encircling the $95 \%$ confidence interval and a box encompassing the interquartile range of the data. Whiskers cover the full range of the data, excluding the outliers. Outliers are marked with crosses.

12 clusters]. This difference is significant $(p=0.0049, z=2.8$, $\mathrm{W}=73, r=0.57$, Wilcoxon signed rank test).

We conclude that pyramidal members of IPP clusters share tuning properties with each other and with their partner in- terneuron. Note that visual tuning properties did not necessarily have to be a priori similar across members of the same IPP cluster, because IPP clusters were defined by analyzing spontaneous patterns of activity in V1 L2/3 in the absence of visual stimulation. 
A

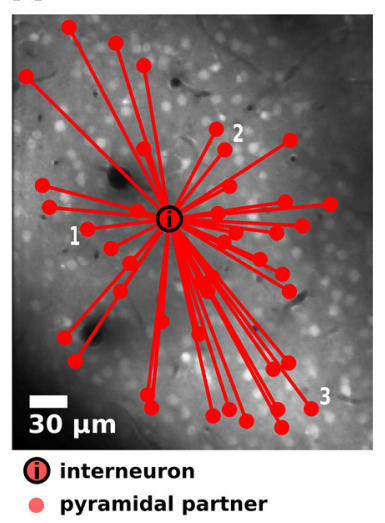

D

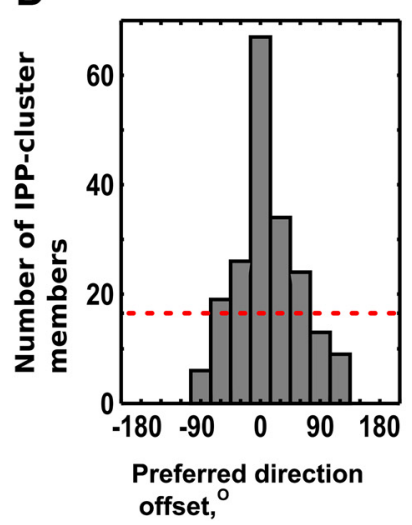

B

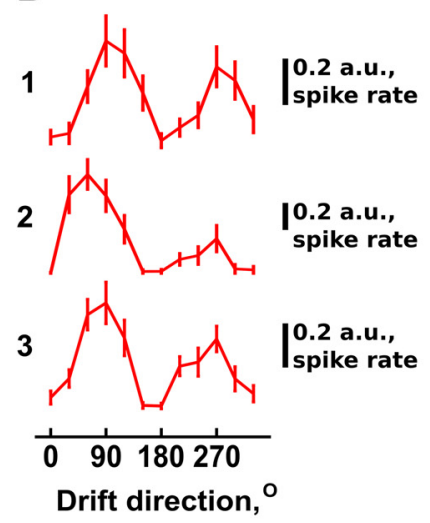

E

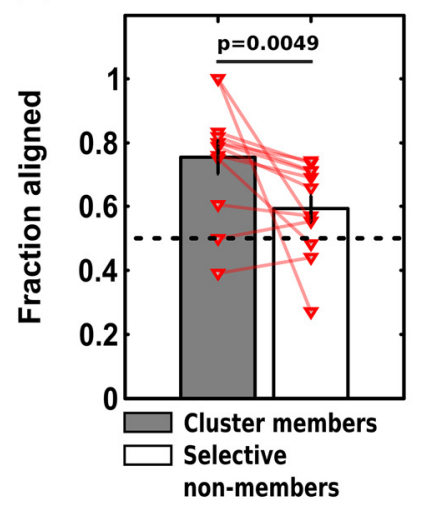

C

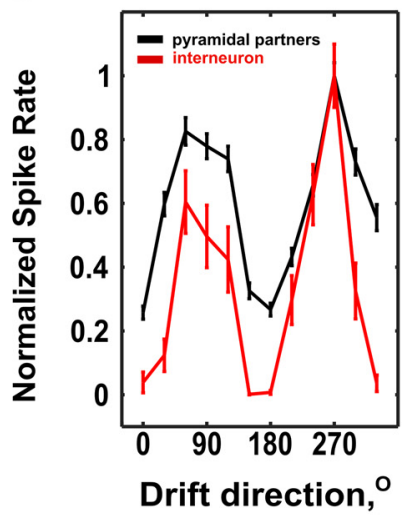

$\mathbf{F}$

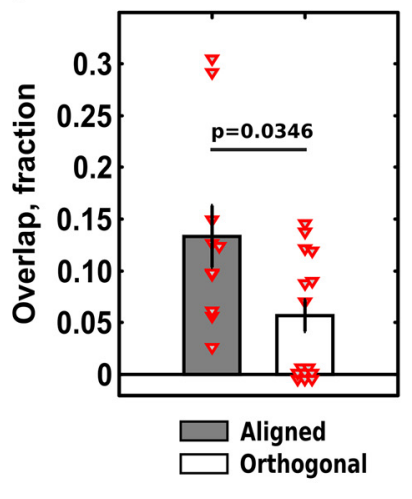

Figure 5. IPP-cluster members have similar functional properties. $\boldsymbol{A}$, Example adult V1 L2/3 IPP-cluster with 45 PC members. The partner interneuron is denoted by "i". $\boldsymbol{B}$, Example tuning functions to drifting gratings for Cells 1-3 shown in $\boldsymbol{A}$. C, Tuning function derived from the pyramidal members of the IPP cluster shown in A (black; see Materials and Methods) compared with the tuning function of the partner interneuron (red). Note that they share high similarity (median Pearson correlation coefficient $\sim 0.58$ over $n=12$ IPP clusters; mean \pm SEM: $0.48 \pm 0.11$ ). $\boldsymbol{D}$, Distribution of differences in the preferred direction of motion between tuned IPP cluster PC members and their partner interneuron, whose preferred direction is by convention set to zero (see Materials and Methods). The majority of tuned IPP cluster members have the same preferred direction as their partner interneuron. Red dotted line, Fraction expected by chance. $\boldsymbol{E}$, Gray Bar, Fraction of IPP cluster PCs whose preferred direction is within $\pm 45^{\circ}$ of the preferred directions of their partner interneuron. White Bar, Control (tuned pyramidal nonmember cells from the same FOV). Black dotted line, Fraction expected by chance. The difference is significant (Wilcoxon signed rank test, $p=0.0049, z=2.8, \mathrm{~W}=73, r=0.57, n=12$ IPP clusters). Data are expressed as mean \pm SEM; cluster members: $0.754 \pm 0.052$, oriented non-members: $0.59 \pm 0.042$. Red triangles denote individual interneuron values; data points for the same interneuron are connected with solid red lines. $\boldsymbol{F}$, IPP clusters with similar (within $\pm 45^{\circ}$ ) tuning ("aligned") show significantly larger PC overlap compared with IPP clusters with "orthogonal" ( $\left.> \pm 45^{\circ}\right)$ preferences, which have very small overlap. Gray Bar, Aligned clusters: $0.133 \pm 0.03$ ( $n=10$ IPP cluster pairs). White Bar, Orthogonal clusters, $0.057 \pm 0.015$ ( $n=16$ IPP-cluster pairs). Statistical significance was determined by the Wilcoxon rank sum test across cluster pairs ( $p=0.0346, z=2.11, W=175, r=0.41$ ). Note that more than half of the orthogonal cluster pairs had no common members, whereas all aligned cluster pairs had at least one shared member.

The fact that they are suggests that IPP clusters have functional significance for the encoding of visual stimuli. In accordance with this, shared nodes preferentially link IPP clusters with similar tuning properties. Figure $5 F$ shows that IPP clusters with similar direction preference (within $45^{\circ}$ of each other) had on average $\sim 13 \%$ shared nodes $[0.133 \pm 0.03$ (fraction), mean \pm SEM, $n=$ 10 cluster pairs], whereas IPP clusters with orthogonal preference (difference $>45$ degrees) shared only $\sim 6 \%$ of nodes $[0.057 \pm$ 0.02 (fraction), mean $\pm \mathrm{SEM}, n=16$ cluster pairs; Fig. $5 F ; p=$ $0.0346, z=2.11, \mathrm{~W}=175, r=0.41$, Wilcoxon rank sum test). Therefore IPP clusters with similar feature-preference retain stronger connectivity through shared high-degree nodes, whereas IPP clusters with orthogonal tuning largely separate from each other in the adult neocortex.

\section{Discussion}

Understanding how neocortical neurons organize into functional groups that multiplex with each other to process information is an open question in systems neuroscience. Pairwise connectivity is not uniform in the neocortex (Song et al., 2005;
Yoshimura et al., 2005; Silberberg and Markram, 2007; Bonifazi et al., 2009; Perin et al., 2011; Kwan and Dan, 2012; Ko et al., 2013; Cossart, 2014; Jiang et al., 2015; Shimono and Beggs, 2015; Karnani et al., 2016). In mouse area V1 pyramidal neurons share stronger physical and functional connections with each other if they are similarly tuned, co-activated by the same type of visual stimulus or receive shared excitatory input from other cortical layers (Gilbert and Wiesel, 1989; Yoshimura et al., 2005; Ko et al., 2011). A body of observations also supports the existence of interneuron-PC partnerships built on functional specificity. For example, fast-spiking inhibitory neurons display specificity in their connections with local PCs by providing the strongest feedback inhibitory synapses to PCs that provide them with the strongest excitatory inputs (Yoshimura and Callaway, 2005; Znamenskiy et al., 2018). Still, how multineuronal ensembles of neurons coordinate to form computationally meaningful units remains incompletely understood.

By analyzing the patterns of firing of individual neurons during epochs of spontaneous activity (Luczak et al., 2007; Ringach, 
2009; Miller et al., 2014; Carrillo-Reid et al., 2015, 2016) in layer $2 / 3$ of mouse area V1 we identified PCs whose firing tends to immediately precede the firing of a neighboring interneuron with high statistical significance (Fig. 2). The interneuron with its pyramidal partners forms an IPP cluster. IPP cluster members have higher probability to fire in synchrony with each other, even when we exclude epochs during which they might be linked via the firing of their partner interneuron (Fig. 3). They are also likely to be inhibited together by the firing of their partner interneuron, if for no other reason, because of the fact that interneurons typically contribute a high density of inputs to PCs located in their vicinity (Gupta et al., 2000; Fino and Yuste, 2011; Packer and Yuste, 2011; Karnani et al., 2014; Rikhye et al., 2017; Safari et al., 2017).

IPP clusters were identified purely based on the temporal structure of individual neuronal events during epochs of spontaneous activity, in the absence of visual stimulation. Despite this, pyramidal members of a specific IPP cluster share direction preference between themselves and with their partner interneuron (Fig. 5), strongly supporting the idea that they have functional significance. This is further corroborated by the fact that pyramidal members of IPP clusters exhibit stronger pairwise functional connectivity with each other than with members of other IPP clusters, suggesting that they form a functionally cohesive group. Co-activated pyramidal units are more efficient at transmitting "units" of information to downstream targets (Smyrnakis and Smirnakis, 2013). They also need to be inhibited together to unambiguously designate the end of information transmission. The need of functionally similar IPP-cluster members to be comodulated together suggests the possibility that the IPP cluster functions as a basic computational unit, the synchrony and burst firing of which is regulated by the activation of its partner interneuron.

Remarkably, in our study nearly all identified interneurons (97\%) belonged to an IPP cluster. Labeling of neocortical interneurons in our Dlx $5 / 6$ mice was nonspecific for type: $\sim 98 \%$ of all cortical interneurons were labeled, including $\mathrm{PV}+, \mathrm{SST}+$, VIP + cells, and a diverse population of interneurons that do not express any of these markers, such as reelin-expressing neurogliaform cells (Stühmer et al., 2002; Monory et al., 2006; Rossignol, 2011; Miyoshi et al., 2010). Thus, IPP clusters appear to be formed by all major types of interneurons, regardless of their functional properties or connectivity pattern, including with other interneurons (for review, see Kepecs and Fishell, 2014; Jiang et al., 2015). This reinforces the notion that IPP clusters may represent a fundamental feature of cortical organization, with different types of clusters playing different computational roles. The computational roles performed by individual IPP clusters will in part reflect the functional and physiological diversity of their partner interneurons.

One possible pitfall to the hypothesis that there is specific regulation of individual IPP clusters by the firing of their partner interneurons is that interneurons typically contribute a high density of inputs to PCs located in their vicinity (Gupta et al., 2000; Olah et al., 2009; Fino and Yuste, 2011; Packer and Yuste, 2011; Karnani et al., 2014; Rikhye et al., 2017; Safari et al., 2017). This dense connectivity is referred to as "blanket inhibition", as an individual $\mathrm{PV}+, \mathrm{SOM}+$, or neurogliaform interneuron typically supplies inhibitory drive to $85-100 \%$ of PCs in its vicinity (within $\sim 100-200 \mu \mathrm{m}$ ). As a result, an individual PC might well receive synapses from every local interneuron. However, the specificity still may be achieved by adjusting the strengths of individual synapses. For example, fast-spiking interneurons form a few strong synapses with PCs that provide the strongest reciprocal excitation, whereas the rest of their PC synapses remain much weaker (Znamenskiy et al., 2018). Combining dense inhibitory connectivity with appropriately graded synaptic strength may help the individual $\mathrm{PV}+$ or SOM+ interneurons to selectively control their pyramidal partners, while at the same time exerting little control over pyramidal belonging to different IPP clusters.

Spontaneous activity patterns suggest that the L $2 / 3$ network in area V1 conforms to a small-world architecture (Fig. 1G,H). At the spatial scale to which we examined the system $(\sim 250 \mu \mathrm{m})$, IPP clusters can be tentatively identified as small-worlds interconnected by a relatively small number of shared units (units that overlap between distinct IPP clusters). Shared PCs preferentially link IPP clusters with similar properties (Fig. $5 F$ ), leading to the relative integration of similarly tuned, versus the relative segregation of orthogonally-tuned clusters. A picture then emerges according to which, under appropriate conditions, computationally relevant IPP clusters can interact via shared cells to form functionally significant super-groups, yoked together for the computation of specific properties (like feature detection) or, perhaps, for the performance of specific logical operations.

This network structure of IPP clusters is not hardwired at birth but undergoes substantial refinement during postnatal development (Fig. 4) in agreement with (Golshani et al., 2009; Rochefort et al., 2009; Ko et al., 2013). Our data show that from before eye opening to juvenile adulthood IPP cluster size and overlap between IPP clusters decreases, the degree of functional connectivity per IPP cluster pyramidal member decreases, and V1 layer $2 / 3$ network architecture increasingly evolves into a higher number of interacting but distinct small-world IPP cluster neighborhoods. In agreement with this, $\sim 39 \%$ of IPP cluster pyramidal members link exclusively with a single interneuron in adulthood, compared with only $\sim 7 \%$ before eye opening (Fig. $4 C$ ). Overall, the number of interneurons one layer $2 / 3$ pyramidal neuron is on average linked with drops from $\sim 4$ before eye opening to $\sim 2$ in adulthood (Fig. $4 D$ ). This is consistent with prior results reporting downscaling of pairwise correlations between neurons during postnatal development (Golshani et al., 2009; Rochefort et al., 2009), and the selective weakening and removal of synaptic connections between disparately tuned pyramidal neurons (Ko et al., 2013).

In summary, $\mathrm{L} 2 / 3$ of area $\mathrm{V} 1$ obeys a principle of organization where PCs are grouped together into feature-selective subnetworks (IPP clusters or cliques; Luce and Perry, 1949), whose firing is linked to the firing of specific, functionally similar cortical interneurons. This notion is intuitively appealing: a group of neurons representing a temporally coherent computational unit ought to be inhibited and disinhibited together, thus providing an intrinsic cortical mechanism for parsing the output of similarly tuned PC populations to higher level areas (Sirota and Buzsáki, 2005; Buzsáki, 2010; Roux and Buzsáki, 2015). Because at least some IPP cliques appear to evolve to represent distinct stimulus features, IPP-clique interactions via shared connections may serve to improve the robustness of specific representations and to help process feature conjunctions involving different aspects of the same stimulus, during processes such as low-level perceptual feature-binding (Kenet et al., 2003; Gao et al., 2010; Miller et al., 2014; Carrillo-Reid et al., 2015; Ji et al., 2015). Overall, our observations suggest a principle of modular organization, where IPP clusters serve as basic cortical modules of computation explicitly "centered" on individual interneurons, whereas computationally meaningful super-groups are formed through interactions between these basic modules mediated by shared PCs. 


\section{References}

Abramoff MD, Magalhaes PJ, Ram SJ (2004) Image processing with ImageJ. Biophotonics Int 11:36-42.

Bassett DS, Bullmore ET (2006) Small-world brain networks. Neuroscientist 12:512-523.

Beggs JM, Plenz D (2003) Neuronal avalanches in neocortical circuits. J Neurosci 23:11167-11177.

Bonifazi P, Goldin M, Picardo MA, Jorquera I, Cattani A, Bianconi G, Represa A, Ben-Ari Y, Cossart R (2009) GABAergic hub neurons orchestrate synchrony in developing hippocampal networks. Science 326:1419-1424.

Buzsáki G (2010) Neural syntax: cell assemblies, synapsembles and readers. Neuron 68:362-385.

Carrillo-Reid L, Miller JE, Hamm JP, Jackson J, Yuste R (2015) Endogenous sequential cortical activity evoked by visual stimuli. J Neurosci 35 : 8813-8828.

Carrillo-Reid L, Yang W, Bando Y, Peterka DS, Yuste R (2016) Imprinting and recalling cortical ensembles. Science 353:691-694.

Chao HT, Chen H, Samaco RC, Xue M, Chahrour M, Yoo J, Neul JL, Gong S, Lu HC, Heintz N, Ekker M, Rubenstein JL, Noebels JL, Rosenmund C, Zoghbi HY (2010) Dysfunction in GABA signalling mediates autismlike stereotypies and rett syndrome phenotypes. Nature 468:263-269.

Cossart R (2014) Operational hub cells: a morpho-physiologically diverse class of GABAergic neurons united by a common function. Curr Opin Neurobiol 26:51-56.

Cossell L, Iacaruso MF, Muir DR, Houlton R, Sader EN, Ko H, Hofer SB, Mrsic-Flogel TD (2015) Functional orgnization of excitatory synaptic strength in primary visual cortex. Nature 518:399-403.

Douglas RJ, Martin KA (1991) A functional microcircuit for cat visual cortex. J Physiol 440:735-769.

Eurich CW, Herrmann JM, Ernst UA (2002) Finite-size effects of avalanche dynamics. Phys Rev E Stat Nonlin Soft Matter Phys 66:066137.

Fino E, Yuste R (2011) Dense inhibitory connectivity in neocortex. Neuron 69:1188-1203.

Gao E, DeAngelis GC, Burkhalter A (2010) Parallel input channels to mouse primary visual cortex. J Neurosci 30:5912-5926.

Gilbert CD, Wiesel TN (1989) Columnar specificity of horizontal and cortico-cortical connections in cat visual cortex. J Neurosci 9:2432-2442.

Golshani P, Gonçalves JT, Khoshkhoo S, Mostany R, Smirnakis S, PorteraCailliau C (2009) Internally mediated developmental desynchronization of neocortical network activity. J Neurosci 29:10890-10899.

Gupta A, Wang Y, Markram H (2000) Organizing principles for a diversity of GABAergic interneurons and synapses in the neocortex. Science 287: 273-278.

Humphries MD, Gurney K, Prescott TJ (2006) The brainstem reticular formation is a small-world, not scale-free, network. Proc Biol Sci 273:503511.

Jiang X, Shen S, Cadwell CR, Berens P, Sinz F, Ecker AS, Patel S, Tolias AS (2015) Principles of connectivity among morphologically defined cell types in adult neocortex. Science 350: aac9462.

Ji W, Gămănuţ R, Bista P, D’Souza RD, Wang Q, Burkhalter A (2015) Modularity in the organization of mouse primary visual cortex. Neuron 87 : 632-643.

Kalatsky VA, Stryker MP (2003) New paradigm for optical imaging: temporally encoded maps of intrinsic signal. Neuron 38:529-545.

Karnani MM, Agetsuma M, Yuste R (2014) A blanket of inhibition: functional inferences from dense inhibitory circuit structure. Curr Opin Neurobiol 26:96-102.

Karnani MM, Jackson J, Ayzenshtat I, Tucciarone J, Manoocheri K, Snider WG, Yuste R (2016) Cooperative subnetworks of molecularly similar interneurons in mouse neocortex. Neuron 90:86-100.

Kenet T, Bibitchkov D, Tsodyks M, Grinvald A, Arieli A (2003) Spontaneously emerging cortical representations of visual attributes. Nature 425: 954-956.

Kepecs A, Fishell G (2014) Interneuron cell types are fit to function. Nature 505:318-326.

Kerlin AM, Andermann ML, Berezovskii VK, Reid RC (2010) Broadly tuned response properties of diverse inhibitory neuron subtypes in mouse visual cortex. Neuron 67:858-871.

Ko H, Hofer SB, Pichler B, Buchanan KA, Sjöström PJ, Mrsic-Flogel TD (2011) Functional specificity of local synaptic connections in neocortical networks. Nature 473:87-91.

Ko H, Cossell L, Baragli C, Antolik J, Clopath C, Hofer SB, Mrsic-Flogel TD
(2013) The emergence of functional microcircuits in visual cortex. Nature 496:96-100.

Kwan AC, Dan Y (2012) Dissection of cortical microcircuits by singleneuron stimulation in vivo. Curr Biol 22:1459-1467.

Lago-Fernández LF, Huerta R, Corbacho F, Sigüenza JA (2000) Fast response and temporal coherent oscillations in small-world networks. Phys Rev Lett 84:2758-2761.

Lee WC, Bonin V, Reed M, Graham BJ, Hood G, Glattfelder K, Reid RC (2016) Anatomy and function of an excitatory network in the visual cortex. Nature 532:370-374.

Luce DR, Perry AD (1949) A method of matrix analysis of group structure. Psychometrika 14:95-116.

Luczak A, Barthó P, Marguet SL, Buzsáki G, Harris KD (2007) Sequential structure of neocortical spontaneous activity in vivo. Proc Natl Acad Sci U S A 104:347-352.

Ma WP, Liu BH, Li Y, Huang ZJ, Zhang LI, Tao HW (2010) Visual representations by cortical somatostatin inhibitory neurons- selective but with weak and delayed responses. J Neurosci 30:14371-14379.

Madisen L, Zwingman TA, Sunkin SM, Oh SW, Zariwala HA, Gu H, Ng LL, Palmiter RD, Hawrylycz MJ, Jones AR, Lein ES, Zeng H (2010) A robust and high-throughput cre reporting and characterization system for the whole mouse brain. Nat Neurosci 13:133-140.

Margrie TW, Brecht M, Sakmann B (2002) In vivo, low-resistance, wholecell recordings from neurons in the anaesthetized and awake mammalian brain. Pflugers Arch 444:491-498.

Markov NT, Ercsey-Ravasz M, Van Essen DC, Knoblauch K, Toroczkai Z, Kennedy H (2013) Cortical high-density counterstream architectures. Science 342: 1238406

Mesik L, Ma WP, Li LY, Ibrahim LA, Huang ZJ, Zhang LI, Tao HW (2015) Functional response properties of VIP-expressing inhibitory neurons in mouse visual and auditory cortex. Front Neural Circuits 9:22.

Meunier D, Lambiotte R, Bullmore ET (2010) Modular and hierarchically modular organization of brain networks. Front Neurosci 4:200.

Miller JE, Ayzenshtat I, Carrillo-Reid L, Yuste R (2014) Visual stimuli recruit intrinsically generated cortical ensembles. Proc Natl Acad Sci U S A 111:E4053-E4061.

Miyoshi G, Hjerling-Leffler J, Karayannis T, Sousa VH, Butt SJ, Battiste J, Johnson JE, Machold RP, Fishell G (2010) Genetic fate mapping reveals that the caudal ganglionic eminence produces a large and diverse population of superficial cortical interneurons. J Neurosci 30:1582-1594.

Monory K, Massa F, Egertova M, Eder M, Blaudzun H Westenbroek R, Kelsch W, Jacob W, Marsch R, Ekker M, Long J, Rubenstein JL, Goebbels S, Nave KA, During M, Klugmann M, Wolfel B, Dodt HU, Zieglgansberger W, Wotjak CT, Mackie K, et al. (2006) The endocannabinoid system controls key epileptogenic circuits in the hippocampus. Neuron 51:455-466.

Ohki K, Chung S, Ch'ng YH, Kara P, Reid RC (2005) Functional imaging with cellular resolution reveals precise micro-architecture in visual cortex. Nature 433:597-603.

Olah S, Füle M, Komlósi G, Varga C, Báldi R, Barzó P, Tamás G (2009) Regulation of cortical microcircuits by unitary gaba-mediated volume transmission. Nature 461:1278-1281.

Packer AM, Yuste R (2011) Dense, unspecific connectivity of neocortical parvalbumin-positive interneurons: a canonical microcircuit for inhibition? J Neurosci 31:13260-13271.

Palagina G, Meyer JF, Smirnakis SM (2017) Complex visual motion representation in mouse area V1. J Neurosci 37:164-183.

Perin R, Berger TK, Markram H (2011) A synaptic organizing principle for cortical neuronal groups. Proc Natl Acad Sci U S A 108:5419-5424.

Rikhye RV, Hu M, Yildirim M, Sur M (2017) Reliable sensory processing in mouse visual cortex through inhibitory interactions between somatostatin and parvalbumin interneurons. bioRxiv. Advance online publication. Retrieved September 11, 2017. doi: 10.1101/187062.

Ringach DL (2009) Spontaneous and driven cortical activity: implications for computation. Curr Opin Neurobiol 19:439-444.

Rochefort NL, Garaschuk O, Milos RI, Narushima M, Marandi N, Pichler B, Kovalchuk Y, Konnerth A (2009) Sparsification of neuronal activity in the visual cortex at eye-opening. Proc Natl Acad Sci U S A 106:1504915054.

Rochefort NL, Narushima M, Grienberger C, Marandi N, Hill DN, Konnerth A (2011) Development of direction selectivity in mouse cortical neurons. Neuron 71:425-432. 
Rossignol E (2011) Genetics and function of neocortical GABAergic interneurons in neurodevelopmental disorders. Neural Plast 2011:649325.

Roux L, Buzsáki G (2015) Tasks for inhibitory interneurons in intact brain circuits. Neuropharmacology 88:10-23.

Rudy B, Fishell G, Lee S, Hjerling-Leffler J (2011) Three groups of interneurons account for nearly $100 \%$ of neocortical GABAergic neurons. Dev Neurobiol 71:45-61.

Runyan CA, Schummers J, Van Wart A, Kuhlman SJ, Wilson NR, Huang ZJ, Sur M (2010) Response features of parvalbumin-expressing interneurons suggest precise roles for subtypes of inhibition in visual cortex. Neuron 67:847-857.

Safari MS, Mirnajafi-Zadeh J, Hioki H, Tsumoto T (2017) Parvalbuminexpressing interneurons can act solo while somatostatin-expressing interneurons act in chorus in most cases on cortical pyramidal cells. Sci Rep $7: 12764$.

Shimono M, Beggs JM (2015) Functional clusters, hubs, and communities in the cortical microconnectome. Cereb Cortex 25:3743-3757.

Silberberg G, Markram H (2007) Disynaptic inhibition between neocortical pyramidal cells mediated by martinotti cells. Neuron 53:735-746.

Sirota A, Buzsáki G (2005) Interaction between neocortical and hippocampal networks via slow oscillations. Thalamus Relat Syst 3:245-259.

Smyrnakis I, Smirnakis S (2013) Information transfer through stochastic transmission of a linear combination of rates. Neural Comput 25:2265-2302.

Song S, Sjöström PJ, Reigl M, Nelson S, Chklovskii DB (2005) Highly nonrandom features of synaptic connectivity in local cortical circuits. PLoS Biol 3:e68.

Sporns O (2011) Networks of the brain. Cambridge, MA: MIT.

Sporns O, Honey CJ (2006) Small worlds inside big brains. Proc Natl Acad Sci U S A 103:19219-19220.
Stosiek C, Garaschuk O, Holthoff K, Konnerth A (2003) In vivo two-photon calcium imaging of neuronal networks. Proc Natl Acad Sci U S A 100:7319-7324.

Stühmer T, Puelles L, Ekker M, Rubenstein JL (2002) Expression from a dlx gene enhancer marks adult mouse cortical GABAergic neurons. Cereb Cortex 12:75-85.

Uhlhaas PJ, Pipa G, Lima B, Melloni L, Neuenschwander S, Nikolić D, Singer W (2009) Neural synchrony in cortical networks: history, concept and current status. Front Integr Neurosci 3:17.

Vogelstein JT, Packer AM, Machado TA, Sippy T, Babadi B, Yuste R, Paninski L (2010) Fast non-negative deconvolution for spike train inference from population calcium imaging. J Neurophysiol 104:3691-3704.

Wang Q, Burkhalter A (2007) Area map of mouse visual cortex. J Comp Neurol 502:339-357.

Watts DJ, Strogatz SH (1998) Collective dynamics of small-world networks. Nature 393:440-442.

Wertz A, Trenholm S, Yonehara K, Hillier D, Raics Z, Leinweber M, Szalay G, Ghanem A, Keller G, Rózsa B, Conzelmann KK, Roska B (2015) Singlecell initiated monosynaptic tracing reveals layer-specific cortical network modules. Science 349:70-74.

Yoshimura Y, Callaway EM (2005) Fine-scale specificity of cortical networks depends on inhibitory cell type and connectivity. Nat Neurosci 8:1552-1559.

Yoshimura Y, Dantzker JL, Callaway E (2005) Excitatory cortical neurons form fine-scale functional networks. Nature 433:868-873.

Znamenskiy P, Kim MH, Muir DR, Iacaruso MF, Hofer SB, Mrsic-Flogel TD (2018) Functional selectivity and specific connectivity of inhibitory neurons in primary visual cortex. bioRxiv. Advance online publication. Retrieved April 4, 2018. doi: 10.1101/294835. 